\title{
Analysis of Regulatory T-cells-specific mRNAs for Improving Prognosis, Immunotherapy Response and Therapeutic Resistance of Patients With Prostate Cancer
}

\section{Mingyi Ju}

China Medical University Department of Social Sciences

Jingyi Fan

China Medical University

Yuanjiang Zou

China Medical University

Mingjie Yu

China Medical University

Longyang Jiang

China Medical University

Qian Wei

China Medical University

Jia Bi

China Medical University

Baohui Hu

China Medical University

Lin Wang

China Medical University

Qiutong Guan

China Medical University

\section{Xinyue Song}

China Medical University

Mingyan Dong

China Medical University

Lifeng Yu

China Medical University

\section{Yan Wang}

China Medical University

Minjie Wei

China Medical University 


\section{Hui Kang}

China Medical University

\section{Wei Xin}

China Medical University

\section{Lin Zhao ( $\nabla$ zl_cmu@163.com)}

China Medical University

\section{Research}

Keywords: Prostate cancer, Regulatory T-cells, Cancer immunotherapy, Therapeutic resistance, Prognostic signature

Posted Date: July 8th, 2021

DOl: https://doi.org/10.21203/rs.3.rs-676662/v1

License: (c) (i) This work is licensed under a Creative Commons Attribution 4.0 International License. Read Full License 


\section{Abstract}

Background: Prostate cancer recognized as a "cold" tumor has an immunosuppressive microenvironment in which regulatory T-cells (Tregs) usually represent a major role. Therefore, identifying a prognostic signature of Tregs has promising benefits of improving survival of prostate cancer patients. However, the prognostic signature based on Tregs-specific mRNAs for prostate cancer is lacking.

Methods: We systematicly analyzed transcriptional profiles of Tregs and 19 other immune cell types using 42 purified immune cell datasets from GEO to identify Tregs-specific mRNAs, and develop and validate a prognostic signature of Tregs (named "TILTregSig") for monitoring prognosis of prostate cancer using TCGA and ICGC datasets. We also applied the signature to five immunotherapy response datasets and GSCALite to analyze the potential of the TILTregSig for predicting CIT response and cancer therapeutic resistance.

Results: We develop the TILTregSig comprising five mRNAs (SOCS2, EGR1, RRM2, TPP1 and C11orf54) for prostate cancer patients. We find that the TILTregSig is a stronger predictor for tumor immunity compared with tumor mutation burden (TMB) and glycolytic activity which have been reported as immune predictors. Further analyses indicate that the TILTregSig may influence tumor immunity mainly by mediating tumor-infiltrating Tregs. Moreover, the TILTregSig also shows promising potential for predicting cancer immunotherapy (CIT) response and therapeutic resistance in multiple cancers.

Conclusions: Our study has highlighted the value of the TILTregSig as a prognostic biomarker of prostate cancer from a tumor-infiltrating Tregs perspective, and strengthened its potential application as predictor of CIT response and cancer therapeutic resistance, which warrants further.

\section{Background}

Prostate cancer is the most frequently diagnosed cancer and the second cause of cancer death in men worldwide, with an estimated incidence of 1,414,259 new cases in 2020 , accounting for $7.3 \%$ of new cancer cases in men (1). Radical prostatectomy (RP) and radiation therapy (RT) are the most common primary treatment options for prostate cancer patients and can provide definitive cure in many patients. Unfortunately, recurrent prostate cancer following primary therapy is common (2), with the incidence of biochemical recurrence (BCR) ranging from $19-35 \%$ at 10 years following RP and around $30 \%$ following RT (3). Therefore, excavating a biomarker that can predict the recurrence holds the promise of improving survival for prostate cancer patients.

Recently, a growing body of evidence has revealed the attractive clinical efficacy of cancer immunotherapy (CIT) in the treatment of prostate cancer. For example, sipuleucel-T has been evaluated in the multicenter Immunotherapy for Prostate Adenocarcinoma Treatment (IMPACT trial) which has been approved by sipuleucel-T's Federal Drug Administration, in addition to three Phase III clinical trials (NCT00065442, NCT00005947, and NCT01133704). Sipuleucel-T-treated patients tended to have a 3-fold increase in activated $\mathrm{T}$ cells in prostatectomy specimens compared to patients who did not receive 
sipuleucel-T (4). Furthermore, the median survival of patients who received sipuleucel-T was 25.8 months, while 21.7 months in placebo-treated patients. Beer TM et al. found that there was a significant difference seen in PFS between patients treated with ipilimumab and patients treated with placebo, 5.6 months in the ipilimumab group compared to 3.8 months in the placebo group (5). Despite the sustained clinical efficacy of CIT, however, only a fraction of patients benefit from them (6). Therefore, it has become a primary priority to excavate a biomarker that can accurately predict the prognosis and response to $\mathrm{CIT}$ for prostate cancer, which will bring tremendous value in guiding the management of prostate cancer patients. Previous studies have unveiled some indicators associated with CIT response such as tumor mutation burden (TMB) (7), eosinophilic count (8), PD-L1 expression (9), deep sequencing of T-cell receptor DNA (10), glycolytic activity (11). However, accurate biomarkers for predicting clinical outcome and CIT responses for prostate cancer patients continue to be largely unexplored.

Prostate cancer defined as a "cold" tumor has an immunosuppressive microenvironment. Tumorinfiltrating lymphocytes (TILs) usually restrain the activity of T-effector cells, which may contribute to cancer progression. Previous study found that TILs resided in prostate cancer tissues was skewed towards regulatory T-cells (Tregs) and T helper 17 (Th17) phenotypes, which suppressed autoreactive T cells and antitumor immune responses (12). In TILs, Tregs are numerous and highly activated $(13,14)$, and are supposed to represent a major mechanism of tumor-induced immune suppression $(15,16)$. Not only do Tregs inhibit T-effector cells, but they appear to fundamentally alter the entire immune milieu related to the tumor. A large body of evidence indicated that tumor-infiltrating Tregs were strongly associated with the response of CIT, and represented a potentially important target for CIT $(15,17)$. In addition, Tregs have been observed significantly associated with poor prognosis of prostate cancer (18, 19). Transcriptional characteristics have recently been found have predictive power for the infiltrations of Tregs, thus leading to the identification of gene expression biomarkers for quantitative evaluation of Tregs and prognosis and CIT response stratifications $(20,21)$. However, Tregs-specific mRNAs, and their application in evaluating Tregs and predicting prognosis and CIT responses, has been not explored.

Therefore, in this study, we systematicly analyzed transcriptional profiles of Tregs and 19 other immune cell types using 42 purified immune cell datasets from GEO to identify Tregs-specific mRNAs and develop a prognostic signature of Tregs (named "TILTregSig") for monitoring prognosis of prostate cancer. The potential of the TILTregSig to serve as a predictive biomarker of CIT response and cancer therapeutic resistance was also explored.

\section{Methods}

\section{Patient data}

Clinical information and transcriptional profiles of prostate cancer patients were retrieved from the Cancer Genome Atlas data portal (TCGA, http://cancergenome.nih.gov/). After the removal of patients with shorter than one month survival time from the data, 454 prostate cancer patients and their corresponding RNA sequencing (RNA-seq) data profiled expressed as transcripts per million (TPM) were 
obtained from TCGA database. R package 'edgeR' was utilized to normalize and process the data by using $\mathrm{R}$ version 4.0.4 software.

For validation, we also downloaded clinical information and transcriptional data of 25 prostate cancer patients (PRAD-FR cohort) from the International Cancer Genome Consortium (ICGC, https://dcc.icgc.org/).

We downloaded the immunotherapy response datasets from the GEO database (GSE19423, GSE111636, GSE67501, GSE53922) and Miao D, et al. (22). The list of these immunotherapy response datasets was displayed in Table S1.

\section{Purified immune cell data}

Transcriptional profiles of T regulatory (Treg) cells and 19 other immune cell types conducted by GPL571 (Affymetrix Human Genome U133A 2.0 Array) were obtained from the publicly available GEO database (https://www.ncbi.nlm.nih.gov/geo/) including GSE38043, GSE11292, GSE22501, GSE22045, GSE23332, GSE65010, GSE43769, GSE50175, GSE42058, GSE59237, GSE233371, GSE37750, GSE14000, GSE4984, GSE27838, GSE8059, GSE46062, GSE17186, GSE39411, GSE50006, GSE13987, GSE85260, GSE56591, GSE16386, GSE16755, GSE13670, GSE11864, GSE84331, GSE51288, GSE44126, GSE26347, GSE142672, GSE134209, GSE101587, GSE66936, GSE49910, GSE28490, GSE93776, GSE67321, GSE72642, GSE28491, GSE28726.

\section{Construction a TILTreg-derived mRNA prognostic signature for prostate cancer}

The mRNA and clinical profiling analysis was developed for identifying TILTregSig (i.e. the TIL-Tregderived prognostic mRNA signature) as follows (Figure S1): (i) Differential expression analysis of mRNAs between Treg-cell lines and other immune cell lines was performed using the R packages 'limma'. Those mRNAs highly expressed in Treg-cell lines and downregulated in other immune cell lines were defined as Tregs-specific mRNAs (by criterion as false discovery rate (FDR) $<0.05$ and LogFC $>2$ ); (ii) To identify mRNAs that were associated with prostate cancer, a list of 384 genes differentially expressed between healthy donors and prostate cancer patients using gene expression profiles excavated from peripheral blood mononuclear cells (PBMCs) of 3 healthy donors and 3 prostate cancer patients (23) were involved in this analysis. Tregs-specific mRNAs overlapped with these 384 genes were extracted out as Tregsspecific mRNAs associated with prostate cancer (TILTreg-associated mRNAs); (iii) Univariate Cox regression analysis was used to explore TILTreg-associated mRNAs related to RFS as prognostic mRNAs; (iv) Multivariate Cox regression analysis was employed to explore candidate biomarkers for monitoring prognosis of prostate cancer (TILTregSig). (v) The corresponding risk scores for patients were calculated according to the expression levels of the genes (expi) and the coefficients of the multivariate Cox regression analysis (bi) for easy application in the clinic. Subsequently, patients were divided into lowand high-risk groups according to the mean risk score. The formula used was as follows: 
Riskscore $=\sum_{i=1}^{n} \operatorname{expi} * b i$

\section{Evaluation of cancer stemness indices.}

Tathiane M Malta et al. applied one-class logistic regression (OCLR) machine-learning algorithm to TCGA datasets to calculate the stemness indices (mRNAsi and mDNAsi) using transcriptomic and epigenetic signatures (24). mDNAsi is reflective of epigenetic features, while mRNAsi is reflective of gene expression. Each stemness index (si) ranges from low (zero) to high (one) stemness.

\section{Genetic variation and methylation analysis of the TILTregSig}

A webtool GSCALite (http://bioinfo.life.hust.edu.cn/web/GSCALite/) was used to analyse the genetic variation and methylation of the genes involved in the TILTregSig. Data in GSCALite were overlapped with the samples derived from TCGA database.

\section{Evaluation of tumor immunity, tumor mutation burden (TMB), glycolysis score and immune-related indicators, and epithelial-mesenchymal transition levels}

In order to assess tumor immunity, we employed Estimation of STromal and Immune cells in MAlignant Tumours using Expression data (ESTIMATE), a method that quantify the immune score, stromal score, ESTIMATE score and tumor purity for each tumor sample that quantifies the immune activity (immune infiltration level) based on the expression of immune genes (25). For each tumor sample, we determined its TMB as the total count of somatic mutations (except silent mutations) detected in the tumor. Glycolysis score, an immune-related signature which has been proved by Zehang Jiang, et al (11), were also estimated in our study for each tumor sample using ssGSEA based on the glycolytic gene set (11) to compare the ability to predict immunity with the TILTregSig. sSGSEA algorithm was utilized to calculate different immune-related indicators (including APC co-inhibiton, APC co-stimulation, chemokine receptors,

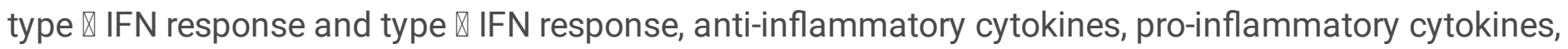
MHC class $\otimes$, cytolytic activity, HLA and TILs) using their feature genes (Table S2). We used epithelialmesenchymal transition (EMT) markers including EMT1, EMT2 and EMT3 which were reported by Sanjeev Mariathasan et al (26) to ssGSEA analysis to evaluate the levels of EMT for prostate cancer patients in different groups.

\section{Evaluation of immune cells infiltrations}

To ensure the accuracy of our results, in this work, we utilized three methods to estimate the immune cell infiltrations in prostate cancer. The first method, we employed sSGSEA to calculate the immune cell infiltration levels using immune cells' marker genes (Table S2). The second method is CIBERSORT 
algorithm (27), which can infer the relative proportions of 22 types of infiltrating immune cells using gene expression profiles obtained from TCGA database. The last method, we employ ImmuneCell Al database (http://bioinfo.life.hust.edu.cn/ImmuCellAl/\#!/) which can evaluate the abundance of 24 immune cells, comprised of 18 T-cell subtypes and 6 other immune cells: B cell, NK cell, Monocyte cell, Macrophage cell, Neutrophil cell and DC cell.

\section{Gene set enrichment analysis}

To explore the potential biological functions of the TILTregSig, we conducted Gene Set Enrichment Analysis (GSEA) based on the curated gene sets "c7.all.v7.4.symbles.gmt [immunologic signature]". Normalized P-value $<0.05$ was considered to be statistical significance.

\section{Statistical analysis}

The expression profiles of mRNAs from TCGA and GEO were shown as raw data and each mRNA was normalized by $\log 2$ transformation for further analysis. The $t$-test $\mathrm{P}<0.05$ was utilized to determine the statistical significance. We calculated the correlation between two variables using the Spearman method. The threshold of $\mathrm{P}<0.05$ (Spearman's correlation test) indicates the significance of correlation. KaplanMeier (K-M) survival curves and log-rank tests were used to compare the survival distribution between the high-risk and low-risk groups via GraphPad Prism version 7.0. To compare the predictive power of different genomic features, time-dependent receiver-operating characteristic (ROC) curves analysis was performed using the R package 'survivalROC', and the area under the ROC curve (AUC) was used to assess the predictive performance of the genomic features. A webtool GSCALite (http://bioinfo.life.hust.edu.cn/web/GSCALite/) was used to analyze the relationships between IC50 data of different molecules and the genes expression levels in the TILTregSig. The Kruskal-Wallis test was used for comparisons among multiple groups. All the statistical analyses were performed in $\mathrm{R}$ version 4.0.4 with additional Bioconductor packages. A two-tailed $\mathrm{P}<0.05$ was considered statistically significant.

\section{Results}

\section{Identification of Tregs-specific mRNAs associated with RFS of prostate cancer.}

To identify Tregs-specific mRNAs, differential expression analysis of mRNAs was performed between Tregs and other immune cell lines, and 2407 dysregulated mRNAs were identified that were highly expressed in Tregs and downregulated in other immune cell lines (false discovery rate $(F D R)<0.05$, LogFC > 2, Table S3). These 2407 dysregulated mRNAs were proposed as Tregs-specific mRNAs. NgarYee Huen et, al. identified 384 genes that were differentially expressed between healthy donors and prostate cancer patients using gene expression profiles excavated from peripheral blood mononuclear cells (PBMCs) of 3 healthy donors and 3 prostate cancer patients (23). To explore mRNAs that were associated with prostate cancer, we further extracted 74 mRNAs out of these 2407 mRNAs which were overlapped with these 384 differentially expressed genes (Table S4). A large body of evidence indicated that recurrent prostate cancer following primary therapy is common with high incidence of BCR (2). 
Therefore, we utilized univariate Cox regression analysis to explore Tregs-specific mRNAs associated with recurrence-free survival (RFS) using gene expression and clinical profiles of 454 patients with prostate cancer from TCGA database. Subsequently, 18 mRNAs were obtained that were significantly associated with RFS of prostate cancer patients (Table S5, P<0.01). In these 18 mRNAs, 11 mRNAs were adverse indicators, while 7 mRNAs were protective indicators for prostate cancer, which were identified significantly associated with RFS and defined as candidate mRNAs of the prognostic signature for prostate cancer.

\section{Construction of a prognostic signature which can predict RFS of prostate cancer.}

To explore a prognostic signature for monitoring RFS of prostate cancer, multivariate Cox regression models were employed using gene expression profiles and clinical information of 454 patients with prostate cancer obtained from TCGA database. Thus, the prognostic signature, named 'TILTregSig', was composed of five Tregs-specific mRNAs (SOCS2, EGR1, RRM2, TPP1 and C11orf54). The risk score system was built as follows: risk score $=(-0.332 \times$ expression value of SOCS2 $)+(-0.111 \times$ expression value of $E G R 1)+(0.286 \times$ expression value of $R R M 2)+(-0.609 \times$ expression value of TPP1 $)+(-0.748 \times$ expression value of $C 11$ orf54). 454 PRAD patients were dichotomized into high- and low-risk groups according to the cut-off value of 0.946 as the median value of risk score.

Kaplan-Meier (K-M) curves revealed that patients in high-risk groups tended to suffer recurrence (Log-rank $P<0.0001$, Fig. 1A). Patients' recurrence rate was increased in high-risk group compared to that in lowrisk group (Fig. 1B). Correlation analysis indicated that RFS status, T, N, Age, cancer status, treatment response and postoperative $\mathrm{RX}$ were significantly associated with the risk score (Fig. $1 \mathrm{C}, \mathrm{P}<0.05)$. The strip chart showed that the risk of patient mortality and recurrence rate obviously increased and the TNM staging gradually rose as the risk score increased (Fig. 1D). The chi-squared test demonstrated that the high-risk group significantly tended to recurrence $(P<0.0001$, Fig. 1E), higher $T$ stage $(P=0.0167$, Fig. 1E), higher $N$ stage $(P<0.0001$, Fig. $1 E)$, older $(P=0.0068$, Fig. $1 E)$, survival with tumor $(P<0.0001$, Fig. $1 E)$, no response to treatment $(P=0.0160$, Fig. $1 E)$ and no postoperative $R X(P<0.0001$, Fig. $1 E)$ compared to the low-risk group of prostate cancer. These results suggested that highly malignant prostate cancer were associated with high-risk score, and our risk score system based on the TILTregSig had tremendous potential to predict RFS for prostate cancer patients.

Cancer stem was proved to be associated with poor prognosis (28). Tathiane M Malta et al. employed OCLR machine-learning algorithm to TCGA datasets to calculate the stemness indices (mRNAsi and mDNAsi) using transcriptomic and epigenetic signatures (24). Thus, we also involved stemness indices derived from study of Tathiane M Malta et al. to validate the clinical association between the TILTregSig and the prognosis of prostate cancer patients. The results showed a significantly positive correlation between the TILTregSig and the stemness indices both in the mRNA expression levels $(P=0.0006, R=$ 0.160 , Figure $S 2 A)$ and DNA methylation levels $(P<0.0001, R=0.296$, Figure $S 2 B)$, which was in consistence with our previous results that high-risk score based on the TILTregSig was markedly associated recurrence of prostate cancer. 


\section{The TILTregSig has robustly predictive value for prostate cancer patients in ICGC dataset.}

To validate the prediction power of the TILTregSig, we further applied the signature to ICGC database. Then, 25 prostate cancer patients were divided into low- and high- risk groups according to the median value of the risk score in ICGC dataset. As shown in Fig. 1F, K-M curves displayed significantly great utility in predicting RFS of prostate cancer. We also found that patients' recurrence rate was increased in highrisk group compared to that in low-risk group (Fig. 1G). The strip chart of clinical characteristics of prostate cancer patients indicated that the risk of mortality and recurrence rate increased as the risk score increased $($ Fig. $1 \mathrm{H})$. The chi-squared test showed that patients in high-risk group markedly tended to suffer recurrence $(P=0.0027$, Fig. 1I). These results illustrates that high-risk score is associated with recurrence of prostate cancer patients, which is in consistence with our previous results in TCGA datatset.

\section{The TILTregSig is an independently prognostic indicator for prostate cancer patients.}

Next, univariate and multivariate Cox regression analysis was carried out to analyze whether the TILTregSig can be an independent predictor for prostate cancer patients. The risk score and other clinicopathological factors were used as covariates. Stage $M$ was not included in this analysis for the reason of only one patient in stage M1 in the TCGA dataset. The results unveiled that the risk score (Multivariate Cox: $\mathrm{HR}=1.170,95 \% \mathrm{Cl}=1.079-1.267 ; \mathrm{P}<0.001$ ), stage $\mathrm{T}$ (Multivariate Cox: $\mathrm{HR}=1.749$, $95 \% \mathrm{Cl}=1.235-2.474 ; \mathrm{P}=0.002$ ) and cancer status (Multivariate Cox: $\mathrm{HR}=8.084,95 \% \mathrm{Cl}=4.557-14.33$; $P<0.001)$ were significantly associated with the RFS and could be seemed as independent RFS prognostic factors for prostate cancer patients (Figure S3A, S3B).

To further investigate the clinical potentiality of the risk score in prostate cancer, stratified analysis based on these clinical characteristics was implemented. The results indicated that the TILTregSig seemed more applicable to predict RFS of prostate cancer patients in the subgroups of T1 and T2, N0, younger than 71, White, response to treatment, did not receive postoperative RX and laterality in bilateral (Figure S3C). Meanwhile, patients in high-risk group had significantly poorer clinical outcomes compared to those in low-risk group. These results illustrate that the TILTregSig can be served as an independently predictor for RFS of prostate cancer patients, and still applicable for patients in some subgroups.

\section{The landscape of genetic variations of the TILTregSig in prostate cancer.}

Genetic alterations have been found usually confer susceptibilities to prostate cancer (29). GSCALite (http://bioinfo.life.hust.edu.cn/web/GSCALite/) is a webtool which can be used to analyse the genetic variation of the genes, and data in the GSCALite were overlapped with the samples derived from TCGA database. Therefore, in order to comprehensively understand the molecular characteristics of the five genes in the TILTregSig, we examined SNV and CNV status of these genes using GSCALite. It was found that the EGR1 (0.6\%) exhibited the highest mutation frequency followed by RRM2 (0.2\%) and TPP1 (0.2\%), while both SOCS2 and C11or54 did not show any mutations in prostate cancer samples (Fig. 2A). In addition, EGR1 had three effective mutation sites, while both RRM2 and TPP1 had one site, respectively (Fig. 2A). Among alteration types, most were focused on the amplification (SOCS2: 7.72\%, RRM2: 4.67\%, 
TPP1: $5.69 \%$ and C11orf54: 7.93\%, Fig. 2B, Table S6), while EGR1 had a widespread frequency of deletion (EGR1: $6.1 \%$, Fig. 2B, Table S6). The investigation of the correlation between CNV and the expression levels of the five genes indicated a significant positive correlation of SOCS2 (Fig. 2C, 2D; Spearman coefficient: $\mathrm{R}=0.18, \mathrm{P}=3.2 \mathrm{e}-04$ ) and TPP1 (Fig. 2C, 2D; Spearman coefficient: $\mathrm{R}=0.19, \mathrm{P}=8.8 \mathrm{e}-05$ ) expression with CNV, which indicated that patients with high expression of SOCS2 and TPP1 were prone to have high CNV load. The above analysis presents a widespread genetic alteration landscape of the five genes in the TILTregSig in prostate cancer patients, suggesting genetic alterations as the molecular mechanism that high-risk score is related to poor prognosis in prostate cancer.

\section{The five mRNAs involved in the TILTregSig have significantly differential methylation between normal and tumor samples in prostate cancer.}

Gene methylation plays a vital role in malignant transformation and can be specific to types of cancers including prostate cancer (30). To get a better understanding of the mechanism of the effect of the genes in TILTregSig on tumorigenesis, we analyzed differential methylation of the five genes using GSCALite. Surprisingly, we found that all of the five genes showed significantly differential methylation between normal and prostate cancer samples ( $\mathrm{P}<0.05$, Fig. 2E). Furthermore, SOCS2 (Fig. 2F, 2G; Spearman coefficient: $\mathrm{R}=-0.61, \mathrm{P}<0.0001$ ), RRM2 (Fig. 2F, 2G; Spearman coefficient: $\mathrm{R}=-0.39, \mathrm{P}<0.0001$ ), TPP1 (Fig. 2F, 2G; Spearman coefficient: $R=-0.21, P=2.3 e-06$ ) and C11orf54 (Fig. 2F, 2G; Spearman coefficient: $R=-0.33, P=4.7 e-14)$ showed significantly negative correlation between gene methylation and expression in prostate cancer, whereas the expression of EGFR (Fig. 2F; P >0.05) showed no significantly correlation with gene methylation. These findings could contribute to enhancing our understanding of the potential mechanisms on the predictive ability of the TILTregSig in prostate cancer.

\section{The TILTregSig is a stronger predictor for tumor immunity in prostate cancer.}

Because the five genes were initially derived from immune cell lines, we consequently investigated whether the signature was related to the tumor immunity. Therefore, we firstly measured the correlation between the TILTregSig and immune-related factors (chemokines, immunoinhibitors, MHCs and receptors), and found that the risk score was commonly associated with these immune-related factors. Especially, the TILTregSig has the highest positive correlation with CCL17 followed by CCL14 among 39 chemokines (Spearman correlation: $C C L 17 . R=0.206, C C L 14 . R=0.202, P<0.0001$, Figure S4). In 24 immunoinhibitors, the TILTregSig presented the highest positive correlation with LGALS9 followed by TGF- $\beta 1$ (Spearman correlation: LGALS9.R $=0.218$, TGF- $\beta 1 . R=0.182, P<0.0001$, Figure S4), while showed the highest negative correlation with CD274 followed by TGFBR1 (Spearman correlation: CD274. $R=-0,185$, TGFBR1.R=-0.175, $P<0.0001$, Figure S4). Moreover, the TILTregSig has the highest negative correlation with B2M among $21 \mathrm{MHCs}$ (Spearman correlation: R=-0.296, P<0.0001, Figure S4), and CXCL1 among 18 receptors (Spearman correlation: $R=-0.244, P<0.0001$, Figure S4). Next, further analysis indicated that type $\otimes$ IFN response and anti-inflammatory cytokines were significantly enhanced in patients with highrisk sores, while type $\otimes$ IFN response was decreased $(P<0.005$, Fig. $3 A)$. 
In light of these results, we further conjectured that the TILTregSig was correlated with tumor immunity and might have potential to predict tumor immunity. To test this hypothesis, we firstly employed the ESTIMATE algorithm to quantify the immune score, stromal score, ESTIMATE score and tumor purity of prostate cancer patients in TCGA dataset. The results showed that the high-risk group significantly tended to have higher immune scores $(P=0.0052$, Fig. 3B, 3C) and ESTIMATE score ( $P=0.0036$, Fig. 3B, $3 C)$ and lower tumor purity $(P=0.0286$, Fig. $3 B, 3 C)$ compared to the low-risk group of prostate cancer patients. However, for stromal score, there was no significant difference between low- and high- risk groups $(P=0.061$, Fig. $3 B, 3 C)$. These results illustrate that the TILTregSig is significantly correlated with tumor immunity, which suggests that the TILTregSig promises to predict tumor immunity in prostate cancer.

TMB (31) and glycolytic activity (11) have been demonstrated to have predictive ability for immune signatures. Next, to compare the predictive ability to tumor immunity, we involved TMB and glycolytic signature in this analysis. ROC curves indicated that the TILTregSig achieved AUC of 0.665 in predicting tumor immune score for prostate cancer, while TME achieved AUC of 0.610 and glycolysis achieved AUC of 0.509 (Fig. 3D). For validating the predictive potential to tumor immunity, we further involved CYT recognized as an immune signature. The TILTregSig achieved AUC of 0.727 in predicting CYT, while TME achieved AUC of 0.626 and glycolysis achieved AUC of 0.459 (Fig. 3E). Our work strongly indicates that the TILTregSig is significantly correlated with tumor immunity and is a stronger predictor than TMB and glycolytic activity for tumor immunity with moderate predictive potential.

\section{The TILTregSig is significantly associated with tumor-infiltrating Tregs in prostate cancer.}

To elucidate the mechanism of the correlation between the TILTregSig and tumor immunity, we further investigated the immune functional annotation using the gene set of "c7.all.v7.4.symbles.gmt [immunologic signature]" by GSEA analysis. The results demonstrated that the TILTregSig is highly associated with many immune cells, such as $C D 8^{+} T$ cells, $C D 4^{+} T$ cells, $B$ cells and Tregs et al. (Fig. 4A). Subsequently, to deeply unveiled the relationship between the TILTregSig and tumor infiltrating immune cells, we then evaluated the infiltration levels of immune cells in high-and low- risk groups in prostate cancer samples using marker genes' expression analysis, CIBERSORT algorithm and ImmuneCell Al database, respectively. We noticed that the consistent results in marker genes' analysis (Fig. 4B), CIBERSORT algorithm (Fig. 4C) and ImmuneCell Al database (Fig. 4D) were that patients with high-risk score had higher infiltrations of Tregs compared to those with low-risk score (Mann-Whitney U test, $\mathrm{P}<$ 0.05, Fig. 4B, 4C, 4D), which suggested a significant correlation between the TILTregSig and Tregs in prostate cancer. For validating these results, we further involved Tregs' marker genes (FoxP3 and TGF- $\beta 1$ ) into our study, and the correlation analysis demonstrated that the TILTregSig was significantly positive related to FoxP3 and TGF- $\beta 1$ expression ( $P<0.05$, Fig. 4E), which was consistent with our previous results (Fig. 4B, 4C, 4D). Additionally, RRM2 showed highest correlation with iTreg cells and nTreg cells among these five genes in the TILTregSig (Fig. 4F), suggesting that RRM2 might be a key gene determined the correlation between the TILTregSig and tumor immunity. These results suggest that the TILTregSig may 
influence tumor immunity mainly by mediating tumor-infiltrating Tregs, and RRM2 may play a vital role in this section, which needs to be verified by further experiments.

\section{The TILTregSig is a powerful predictor for infiltrations of Tregs in prostate cancer.}

In view of the relationships between Tregs and the TILTregSig in the TME, we further conjectured that the TILTregSig had potential for predicting infiltrations of Tregs in prostate cancer. To test this hypothesis, prostate cancer patients in TCGA dataset were firstly split into Treg-low group $(n=227)$ and Treg-High group $(n=227)$ based on the median infiltration levels of Tregs. Principal component analysis (PCA) demonstrated that the TILTregSig was able to distinguish Treg-low cluster from Treg-High cluster (Fig. 4G), which preliminary hinted us that the TILTregSig had the potential of predicting the infiltrations of Tregs in the prostate cancer.

Additionally, we found that patients in Treg-high group had significantly higher risk scores compared to those in Treg-low group $(P<0.001$, Fig. $4 H)$. Particularly, the expression of SOCS2, EGR1, TPP1 and C11 orf54 was markedly lower in patients with high infiltration levels of Tregs compared to patients with low infiltration levels of Tregs, while RRM2 was markedly higher $(P<0.0001$, Fig. 41$)$. These results reconfirmed that the predictive potential of the TILTregSig for Tregs' infiltrations in prostate cancer.

Therefore, to evaluate the predictive power of the TILTregSig for Tregs' infiltrations, ROC curves were employed in further analysis. Additionally, FoxP3 has been proved as a classic indicator of Tregs (32). Therefore, we also involved FoxP3 in this analysis to compare the predictive ability to the TILTregSig. Surprisingly, we found that the TILTregSig represented high potential as an indicator of Tregs (AUC = 0.897, Fig. $4 \mathrm{~J}$ ), as compared to FoxP3 being a predictor with moderate potential in prostate cancer (AUC $=0.763$, Fig. $4 \mathrm{~J}$ ). Moreover, we further constructed a combined model consist of the TILTregSig and FoxP3, and found that the combined model had weaker predictive power compared with the TILTregSig (AUC $=0.767$, Fig. $4 \mathrm{~J})$. These results suggest that the TILTregSig is a robust and accurate predictor for Tregs in prostate cancer, and its predictive power is stronger than FoxP3.

\section{The TILTregSig has predictive potential as an indicator of response to CIT.}

Accumulated evidence demonstrated patients with low infiltrations of Tregs presented a durable clinical response to CIT (33). Our previous data demonstrated that the TILTregSig was associated with Tregs, suggesting that the TILTregSig might be a pivotal factor that mediated the clinical response to CIT. The correlation between our TILTregSig and check-point genes expression indicated that the risk score was markedly correlated with check-point genes expression, and most were positively correlated $(P<0.05$, Fig. 5A). Subsequently, for investigated whether the TILTregSig could predict patients' response to CIT, we utilized five CIT response associated datasets (GSE19423, GSE111636, GSE67501, GSE53922 and Miao D, et al. (22)). We found that the significant therapeutic advantages and clinical response to CIT in patients with high-risk score compared to those with low-risk score were confirmed in bladder urothelial carcinoma (BLCA), kidney renal clear cell carcinoma (KIRC), prostate carcinoma (PRCA) and renal cell carcinoma (RCC). However, in bladder adenocarcinoma (BLAD), patients with high-risk score had lower 
immunotherapy response rate compared to those with low-risk score (Fig. 5B). Tregs and TME stroma activity, usually mediated immune tolerance of tumors, was also assessed $(12,34)$. We found that Tregs were significantly activated in tumors with high-risk score in BLAD-GSE19423 dataset, while inhibited in BLCA-GSE111636 and PRCA-GSE53922 datasets $(P<0.05$, Fig. 5C). Additionally, TME stroma activity was significantly enhanced in patients with high-risk sore such as the activation of epithelialmesenchymal transition (EMT) in BLAD-GSE19423 dataset, while was decreased in tumors with high-risk score in BLCA-GSE111636 and PRCA-GSE53922 datasets $(P<0.05$, Fig. 5C). The above data imply that the TILTregSig is correlated with CIT response and might have potential for predicting CIT response. To assess the predictive power of the TILTregSig for CIT response, ROC curves were employed in this work. The results showed that the TILTregSig achieved AUC of $0.710,0.677,0.799,0.614$ and 0.695 for BLADGSE19423, BLCA-GSE111636, KIRC-GSE67501, PRCA-GSE53922 and RCC respectively in predicting the response to CIT, which implied that the TILTregSig was a potential and robust biomarker for response assessment of CIT with moderate predictive potential (Fig. 5D). In summary, our work strongly indicated that the TILTregSig was significantly correlated with immunotherapy response, and the established TILTregSig would contribute to predicting the response to CIT.

\section{The TILTregSig is a promising marker of therapeutic resistance in pan-cancers.}

Considering Treg-cells have been indicated that usually promotes resistance to cancer therapy (35), we investigated whether our TILTregSig was correlated with cancer therapeutic resistance. To unveil the relationship between the TILTregSig and therapeutic resistance, we utilized GSEA analysis. As shown in Fig. 6A, GSEA predicted that the TILTregSig was significantly associated with resistance to different therapies, including salirasib, endocrine therapy, doxorubicin and SB216763 in prostate cancer $(P<0.05$, Fig. 6A). Next, a landscape plot was generated by GSCALite to depict the relationships between the five genes expression in the TILTregSig and drug responses in pan-cancers. The bubble heat map showed that some genes exhibited significant correlations with lower half inhibitory centration (IC50) data. In detail, EGR1, TPP1 and SOCS2 conferred drug resistance, while RRM2 and C11orf54 exhibited drug sensitivity in pan-cancers (Fig. 6B). These results imply the TILTregSig as a promising indicator for therapeutic resistance in pan-cancers.

\section{Discussion}

TILs serve as the cellular underpinnings of cancer immunotherapies, and a better understanding of TILs in the tumor microenvironment (TME) is essential for deciphering mechanisms of immunotherapies, defining predictive biomarkers, and identifying novel therapeutic targets. As a key component of the TILs, Tregs usually play a pivotal role in the tumor development and progression due to their immunosuppressive functions in the TME. Therefore, it would realize better benefits of improving survival of cancer patients, if Tregs can be quantitative evaluation. However, the traditional method of TILs quantification, including making visual measurements through a microscope by pathologists using hematoxylin and eosin- or immunohistochemistry-stained tumor sections, usually suffer from bias and 
variability (36). Therefore, identification of a Tregs-specific signature based on genomic profiles may open up a new path to the prediction for survival and immunotherapies response of cancer patients.

In our study, we develop and validate a prognostic signature (named TILTregSig) comprised of Tregsspecific mRNAs for prostate cancer, which associated with RFS in prostate cancer. Our data show a significantly positive correlation between the TILTregSig and stemness indices both in transcriptomic (mRNA expression levels) and epigenetic (DNA methylation levels) levels. These observations consistently indicate that the TILTregSig is a risk factor for prognosis of prostate cancer.

Cancer is a disease driven by genetic variation and mutation (29), and prostate cancer has been recognized as high intratumoral genetic heterogeneity (37). We also identify the landscape of genetic variations of the five genes in TILTregSig in prostate cancer. The results demonstrate that the EGR1 exhibited the highest mutation frequency followed by RRM2 and TPP1, while both SOCS2 and C11or54 do not show any mutations in prostate cancer samples. Moreover, high expression of SOCS2 and TPP1 are prone to have high CNV load. Previous study indicated that CNV and SNV status was reported to be significantly associated with overall cancer risk and metastasis (37-39). These data suggest genetic alterations as the molecular mechanism that high-risk score is related to poor prognosis in prostate cancer.

In addition, our data show that type $\otimes$ IFN response and anti-inflammatory cytokines are significantly

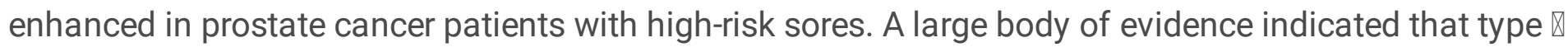
IFN response was emerging as a key driver of immunosuppression and tumor progression (40). Antiinflammatory cytokines are proved that usually involved in cancer progression and related with worse prognosis (41). These previous research are consistent with our findings that prostate cancer patients with high-risk scores have high levels of type $\otimes$ IFN response and anti-inflammatory cytokines, and are related to poor prognosis. This observation has important implications for comprehending the mechanism of the influence of the TILTregSig on anti-tumor immunity.

Moreover, we also unveil that the TILTregSig significantly correlates with tumor immunity. TMB (7) and glycolytic activity (11) have been demonstrated to have promising potential to predict tumor immunity. Subsequently, in comparison with TMB and glycolytic activity, the TILTregSig displays higher predictive power for predicting tumor immunity than TMB and glycolytic activity, with moderate predictive potential. This observation enhances the predictive accuracy of the evaluation of tumor immunity based on existing markers in prostate cancer, which have tremendous significance to improve the prognosis of prostate cancer patients.

Furthermore, the TILTregSig shows higher potential as an indicator of Tregs in prostate cancer with moderate potential, while compared to FoxP3 recognized as a hallmark of Tregs (32). A high infiltration of Tregs is associated with poor survival in various cancers (42). Tregs act on innate immune cells and effector $T$ cells to suppress the anticancer immunity that is mediated by natural killer cells, cytotoxic $\mathrm{CD}^{+} \mathrm{T}$ cells and pro-inflammatory cytokines through secretion of inhibitory cytokines, such as IL-10, 
TGF- $\beta$, and IL-35 (43-45). Moreover, IFN-Is can also enhance the suppressive effects of Tregs, and the IFN-I production in the tumor drives the suppressive Tregs' phenotype (46). Our previous results show that type $\mathbb{I F N}$ response and anti-inflammatory cytokines are significantly enhanced in prostate cancer patients with high-risk sores. Base on above data, we infer that Tregs may affect prostate cancer prognosis through exerting their immunosuppressive functions by regulating type $\mathbb{Q}$ IFN response and the secretion of anti-inflammatory cytokines.

Cancer immunotherapy is a validated and critically important approach for treating patients with cancer. A large body of evidence indicated that Tregs were prevalent in nearly all cancers and, as immunosuppressive regulators of immune responses, they were strongly associated with the response of CIT $(15,47)$. Given the correlation between the TILTregSig and Tregs, we involve five CIT response associated datasets (GSE19423, GSE111636, GSE67501, GSE53922 and Miao D, et al. (22)), and find that the TILTregSig is a promising biomarker for predicting CIT response. Further investigation is therefore warranted to establish the potential utility of the TILTregSig as an additional measure to identify patients likely to respond to CIT. In addition, these findings support the development of agents targeting to tumorinfiltrating Tregs for use in combination with existing CIT. However, it requires further clinical validation using an agent targeting Tregs to be considered predictive of response to this class of agents. As our signature has been derived independently of any specific molecular agent targeting the tumor-infiltrating Tregs, it may have widespread utility of candidate drugs currently in development.

Given Tregs have been indicated usually promoting resistance to therapy (35), we reason that the TILTregSig can be applicable to the prediction of cancer therapeutic resistance. We firstly find that the TILTregSig is significantly associated with resistance to different therapies, including salirasib, endocrine therapy, doxorubicin and SB216763 in prostate cancer. Further analysis unveil that EGR1, TPP1 and SOCS2 confer drug resistance, while RRM2 and C11orf54 exhibit drug sensitivity in pan-cancers. Previous study demonstrate that EGR1 is related with cancer therapy resistance, such as radiation resistance (48) and drug resistance (49). SOCS2 was also proved as a therapeutic resistance-related gene for cancers (50). Altogether, these results indicate the TILTregSig also can be served as a promising marker for therapeutic resistance in cancers. Of course, the association between the TILTregSig and therapeutic resistance revealed in this study needs to be validated in clinical.

Our results present one unexpected finding that patients with BLCA, KIRC, PRCA and RCC in high-risk groups were prone to better response to $\mathrm{CIT}$, while patients with high-risk score had lower $\mathrm{CIT}$ response rate in BLAD. Tregs and TME stroma activity, such as EMT activity, usually induce tumor immune tolerance and relate to low $\mathrm{CIT}$ response rate $(12,34)$. Further investigation shows that Tregs are significantly activated in tumors with high-risk scores in BLAD, while inhibited in BLCA and PRCA. Additionally, EMT activity is significantly enhanced in patients with high-risk scores in BLAD, while decreased in BLCA, KIRC and PRCA. Given these data, we confer that the distinction of CIT response rate in diverse cancers may be on account of the different activations of Tregs and EMT in diverse cancers. 
Despite the significant results obtained in the current study, there are inevitably several shortcomings of our study that should be acknowledged. First, the signature described here may be limited by the decision to only include genes within publicly available datasets, which may introduce bias into the results. Second, transcriptomics analysis only can reflect some aspects of immune status rather than global alterations. Third, the reliability of our results from the bioinformatics analysis is still challenged by the lack of in vitro or in vivo experiments.

\section{Conclusions}

In conclusion, in this work, an integrative analysis of immune, mRNA and clinical profiles has been represented for identifying a Tregs-specific prognostic signature (TILTregSig) for patients with prostate cancer. The TILTregSig display an independently predictive potential for prognosis of prostate cancer patients, even when adjusting for clinical covariates. Further analyses indicate that the TILTregSig may influence tumor immunity mainly by mediating tumor-infiltrating Tregs, and it can be served as a potential indicator for tumor-infiltrating Tregs in prostate cancer. Moreover, we also find that the TILTregSig is capable of assessing CIT response in multiple cancers and shows a promising potential for predicting cancer therapeutic resistance. To the best of our knowledge, this is the first study to excavate a Tregsspecific prognostic signature for prostate cancer, highlighting the impact of Tregs on CIT response and therapeutic resistance, potentially enabling more precise and personalized treatment in the future.

\section{List Of Abbreviations}

Regulatory T-cells: Tregs; tumor mutation burden: TMB; Cancer immunotherapy: CIT; Radical prostatectomy: RP; Radiation therapy: RT; Biochemical recurrence: BCR; Tumor-infiltrating lymphocytes: TILs; The Cancer Genome Atlas data portal: TCGA; Transcripts per million: TPM; The International Cancer Genome Consortium: ICGC; Peripheral blood mononuclear cells: PBMCs; One-class logistic regression: OCLR; Estimation of STromal and Immune cells in MAlignant Tumours using Expression data: ESTIMATE; Epithelial-mesenchymal transition: EMT; Gene Set Enrichment Analysis: GSEA; Kaplan-Meier: K-M; Receiver-operating characteristic: ROC; False discovery rate: FDR; Principal component analysis: PCA; Bladder urothelial carcinoma: BLCA; Kidney renal clear cell carcinoma: KIRC; Prostate carcinoma: PRCA; Renal cell carcinoma: RCC; Bladder adenocarcinoma: BLAD; Epithelial-mesenchymal transition: EMT.

\section{Declarations}

\section{Ethics approval and consent to participate}

Not applicable.

\section{Consent for publication}

Not applicable. 
Availability of data and materials

The authors would also like to thank TCGA (http://cancergenome.nih.gov/), ICGC (https://dcc.icgc.org/) and GEO (https://www.ncbi.nlm.nih.gov/geo/) projects for the data access.

\section{Competing interests}

The authors declare that they have no competing interests.

\section{Funding}

This work was supported by grants from National Natural Science Foundation of China (No. 82073281, 82073884 , U20A20413,81903658), Science and Technology Program of Liaoning Province (2017225036), Shenyang S\&T Projects (No. 19-109-4-09, 20-204-4-22), Program for Shenyang High Level Talent Innovation and Entrepreneurship Team (2019-SYRCCY-B-01), Liaoning Revitalization Talents Program (No. XLYC1807201) and Major Special S\&T Projects in Liaoning Province [2019JH1/10300005].

\section{Authors' contributions}

Conceptualization, Mingyi Ju; Collection and Assembly of Data, Jia Bi, Longyang Jiang and Qian Wei; Data Analysis and Interpretation, Yan Wang, Baohui Hu and Qiutong Guan; Original Draft Preparation, Xinyue Song and Mingyan Dong; Writing, Jingyi Fan, Yuanjiang Zou and Mingjie Yu; Visualization and Supervision, Minjie Wei, Hui Kang, Wei Xin and Lin Zhao.

\section{Acknowledgements}

Not applicable.

\section{Conflict of interest}

The authors have declared no conflicts of interest.

\section{References}

1. Sung H, Ferlay J, Siegel RL. Global cancer statistics 2020: GLOBOCAN estimates of incidence and mortality worldwide for 36 cancers in 185 countries. 2021.

2. May EJ, Viers LD, Viers BR, Kawashima A, Kwon ED, Karnes RJ, et al. Prostate cancer post-treatment follow-up and recurrence evaluation. Abdom Radiol (NY). 2016;41:862-76.

3. Freedland SJ, Humphreys EB, Mangold LA, Eisenberger M, Dorey FJ, Walsh PC, et al. Risk of prostate cancer-specific mortality following biochemical recurrence after radical prostatectomy. JAMA. 2005;294:433-9.

4. Fong L, Carroll P, Weinberg V, Chan S, Lewis J, Corman J, et al. Activated lymphocyte recruitment into the tumor microenvironment following preoperative sipuleucel-T for localized prostate cancer. 
Journal of the National Cancer Institute. 2014;106.

5. Beer TM, Kwon ED, Drake CG, Fizazi K, Logothetis C, Gravis G, et al. Randomized, Double-Blind, Phase III Trial of Ipilimumab Versus Placebo in Asymptomatic or Minimally Symptomatic Patients With Metastatic Chemotherapy-Naive Castration-Resistant Prostate Cancer. Journal of clinical oncology : official journal of the American Society of Clinical Oncology. 2017;35:40-7.

6. Hegde PS, Chen DS. Top 10 Challenges in Cancer Immunotherapy. Immunity. 2020;52:17-35.

7. Chan TA, Yarchoan M, Jaffee E, Swanton C, Quezada SA, Stenzinger A, et al. Development of tumor mutation burden as an immunotherapy biomarker: utility for the oncology clinic. Annals of oncology : official journal of the European Society for Medical Oncology. 2019;30:44-56.

8. Moreira A, Leisgang W, Schuler G, Heinzerling L. Eosinophilic count as a biomarker for prognosis of melanoma patients and its importance in the response to immunotherapy. Immunotherapy. 2017:9:115-21.

9. Patel SP, Kurzrock R. PD-L1 Expression as a Predictive Biomarker in Cancer Immunotherapy. Molecular cancer therapeutics. 2015;14:847-56.

10. Page DB, Yuan J, Redmond D, Wen YH, Durack JC, Emerson R, et al. Deep Sequencing of T-cell Receptor DNA as a Biomarker of Clonally Expanded TILs in Breast Cancer after Immunotherapy. Cancer immunology research. 2016;4:835-44.

11. Jiang Z, Liu Z, Li M, Chen C, Wang X. Increased glycolysis correlates with elevated immune activity in tumor immune microenvironment. EBioMedicine. 2019;42:431-42.

12. Sfanos KS, Bruno TC, Maris CH, Xu L, Thoburn CJ, DeMarzo AM, et al. Phenotypic analysis of prostate-infiltrating lymphocytes reveals TH17 and Treg skewing. Clinical cancer research : an official journal of the American Association for Cancer Research. 2008;14:3254-61.

13. Lavin Y, Kobayashi S, Leader A, Amir E-AD, Elefant N, Bigenwald C, et al. Innate Immune Landscape in Early Lung Adenocarcinoma by Paired Single-Cell Analyses. Cell. 2017;169.

14. De Simone M, Arrigoni A, Rossetti G, Gruarin P, Ranzani V, Politano C, et al. Transcriptional Landscape of Human Tissue Lymphocytes Unveils Uniqueness of Tumor-Infiltrating T Regulatory Cells. Immunity. 2016;45:1135-47.

15. Tanaka A, Sakaguchi S. Regulatory T cells in cancer immunotherapy. Cell Res. 2017;27:109-18.

16. Chao JL, Savage PA. Unlocking the Complexities of Tumor-Associated Regulatory T Cells. Journal of immunology (Baltimore, Md : 1950). 2018;200:415-21.

17. Munn DH, Sharma MD, Johnson TS. Treg Destabilization and Reprogramming: Implications for Cancer Immunotherapy. Cancer research. 2018;78:5191-9.

18. Watanabe M, Kanao K, Suzuki S, Muramatsu H, Morinaga S, Kajikawa K, et al. Increased infiltration of CCR4-positive regulatory $\mathrm{T}$ cells in prostate cancer tissue is associated with a poor prognosis. Prostate. 2019;79:1658-65.

19. Flammiger A, Weisbach L, Huland H, Tennstedt P, Simon R, Minner S, et al. High tissue density of FOXP3+ T cells is associated with clinical outcome in prostate cancer. Eur J Cancer. 2013;49:1273-9. 
20. Pesenacker AM, Chen V, Gillies J, Speake C, Marwaha AK, Sun A, et al. Treg gene signatures predict and measure type 1 diabetes trajectory. JCI Insight. 2019;4.

21. Magnuson AM, Kiner E, Ergun A, Park JS, Asinovski N, Ortiz-Lopez A, et al. Identification and validation of a tumor-infiltrating Treg transcriptional signature conserved across species and tumor types. Proceedings of the National Academy of Sciences of the United States of America. 2018;115:E10672-E81.

22. Miao D, Margolis CA, Gao W, Voss MH, Li W, Martini DJ, et al. Genomic correlates of response to immune checkpoint therapies in clear cell renal cell carcinoma. Science (New York, NY). 2018;359:801-6.

23. Huen N-Y, Pang AL-Y, Tucker JA, Lee T-L, Vergati M, Jochems $C$, et al. Up-regulation of proliferative and migratory genes in regulatory $\mathrm{T}$ cells from patients with metastatic castration-resistant prostate cancer. International journal of cancer. 2013;133:373-82.

24. Malta TM, Sokolov A, Gentles AJ, Burzykowski T, Poisson L, Weinstein JN, et al. Machine Learning Identifies Stemness Features Associated with Oncogenic Dedifferentiation. Cell. 2018;173.

25. Yoshihara K, Shahmoradgoli M, Martínez E, Vegesna R, Kim H, Torres-Garcia W, et al. Inferring tumour purity and stromal and immune cell admixture from expression data. Nature communications. 2013;4:2612.

26. Mariathasan S, Turley SJ, Nickles D, Castiglioni A, Yuen K, Wang Y, et al. TGF $\beta$ attenuates tumour response to PD-L1 blockade by contributing to exclusion of T cells. Nature. 2018;554:544-8.

27. Newman AM, Liu CL, Green MR, Gentles AJ, Feng W, Xu Y, et al. Robust enumeration of cell subsets from tissue expression profiles. Nature methods. 2015;12:453-7.

28. Clarke MF. Clinical and Therapeutic Implications of Cancer Stem Cells. The New England journal of medicine. 2019;380:2237-45.

29. Koboldt DC, Zhang Q, Larson DE, Shen D, McLellan MD, Lin L, et al. VarScan 2: somatic mutation and copy number alteration discovery in cancer by exome sequencing. Genome Res. 2012;22:568-76.

30. Nowacka-Zawisza M, Wiśnik E. DNA methylation and histone modifications as epigenetic regulation in prostate cancer (Review). Oncol Rep. 2017;38:2587-96.

31. Goodman AM, Kato S, Bazhenova L, Patel SP, Frampton GM, Miller V, et al. Tumor Mutational Burden as an Independent Predictor of Response to Immunotherapy in Diverse Cancers. Molecular cancer therapeutics. 2017;16:2598-608.

32. Yang BH, Hagemann S, Mamareli P, Lauer U, Hoffmann U, Beckstette M, et al. Foxp3(+) T cells expressing RORyt represent a stable regulatory T-cell effector lineage with enhanced suppressive capacity during intestinal inflammation. Mucosal Immunol. 2016;9:444-57.

33. Saleh R, Elkord E. Treg-mediated acquired resistance to immune checkpoint inhibitors. Cancer letters. 2019;457:168-79.

34. Chen DS, Mellman I. Elements of cancer immunity and the cancer-immune set point. Nature. 2017;541:321-30. 
35. Oweida A, Hararah MK, Phan A, Binder D, Bhatia S, Lennon S, et al. Resistance to Radiotherapy and PD-L1 Blockade Is Mediated by TIM-3 Upregulation and Regulatory T-Cell Infiltration. Clinical cancer research : an official journal of the American Association for Cancer Research. 2018;24:5368-80.

36. Shaban M, Khurram SA, Fraz MM, Alsubaie N, Masood I, Mushtaq S, et al. A Novel Digital Score for Abundance of Tumour Infiltrating Lymphocytes Predicts Disease Free Survival in Oral Squamous Cell Carcinoma. Sci Rep. 2019;9:13341.

37. Wu B, Lu X, Shen H, Yuan X, Wang X, Yin N, et al. Intratumoral heterogeneity and genetic characteristics of prostate cancer. International journal of cancer. 2020;146:3369-78.

38. Haiman CA, Han Y, Feng Y, Xia L, Hsu C, Sheng X, et al. Genome-wide testing of putative functional exonic variants in relationship with breast and prostate cancer risk in a multiethnic population. PLoS Genet. 2013;9:e1003419.

39. Gallagher S, Hughes E, Wagner S, Tshiaba P, Rosenthal E, Roa BB, et al. Association of a Polygenic Risk Score With Breast Cancer Among Women Carriers of High- and Moderate-Risk Breast Cancer Genes. JAMA Netw Open. 2020;3:e208501.

40. Snell LM, McGaha TL, Brooks DG. Type I Interferon in Chronic Virus Infection and Cancer. Trends in immunology. 2017;38:542-57.

41. Qu X, Tang Y, Hua S. Immunological Approaches Towards Cancer and Inflammation: A Cross Talk. Front Immunol. 2018;9:563.

42. Saito T, Nishikawa H, Wada H, Nagano Y, Sugiyama D, Atarashi K, et al. Two FOXP3(+)CD4(+) T cell subpopulations distinctly control the prognosis of colorectal cancers. Nature medicine. 2016;22:67984.

43. Vignali DAA, Collison LW, Workman CJ. How regulatory T cells work. Nature reviews Immunology. 2008;8:523-32.

44. Ohue Y, Nishikawa H. Regulatory $\mathrm{T}$ (Treg) cells in cancer: Can Treg cells be a new therapeutic target? Cancer Sci. 2019;110:2080-9.

45. Seruga B, Zhang H, Bernstein LJ, Tannock IF. Cytokines and their relationship to the symptoms and outcome of cancer. Nature reviews Cancer. 2008;8:887-99.

46. Stewart CA, Metheny H, lida N, Smith L, Hanson M, Steinhagen F, et al. Interferon-dependent IL-10 production by Tregs limits tumor Th17 inflammation. J Clin Invest. 2013;123:4859-74.

47. Moreno Ayala MA, Li Z, DuPage M. Treg programming and therapeutic reprogramming in cancer. Immunology. 2019;157:198-209.

48. Cook PJ, Thomas R, Kingsley PJ, Shimizu F, Montrose DC, Marnett LJ, et al. Cox-2-derived PGE2 induces Id1-dependent radiation resistance and self-renewal in experimental glioblastoma. Neurooncology. 2016;18:1379-89.

49. Lopez-Ayllon BD, Moncho-Amor V, Abarrategi A, Ibañez de Cáceres I, Castro-Carpeño J, Belda-Iniesta $C$, et al. Cancer stem cells and cisplatin-resistant cells isolated from non-small-lung cancer cell lines constitute related cell populations. Cancer medicine. 2014;3:1099-111. 
50. von Manstein V, Yang CM, Richter D, Delis N, Vafaizadeh V, Groner B. Resistance of Cancer Cells to Targeted Therapies Through the Activation of Compensating Signaling Loops. Curr Signal Transduct Ther. 2013;8:193-202.

\section{Figures}

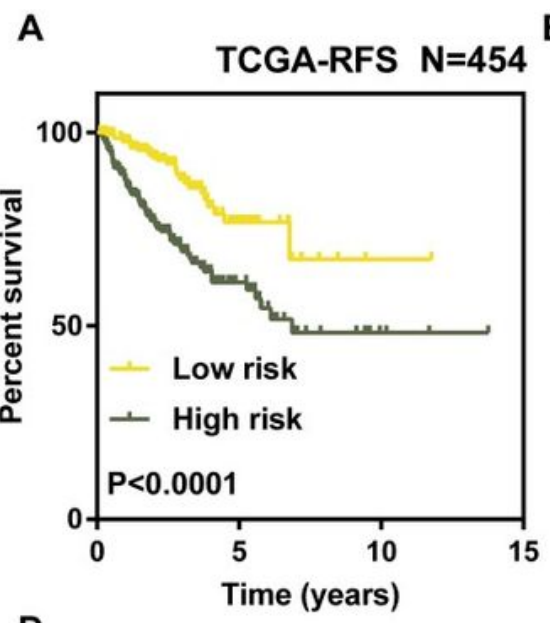

D

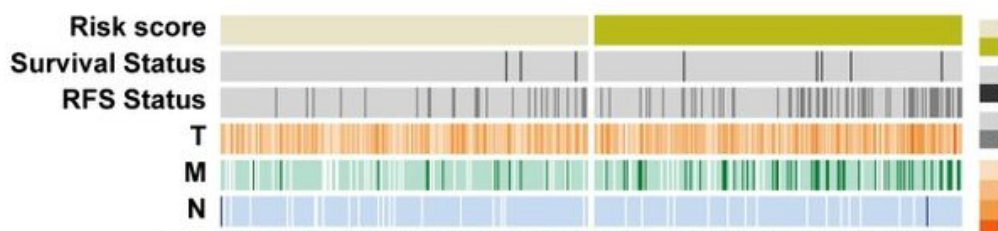

Cancer Status

Race

Treatment Response

Postoperative RX Laterality

E

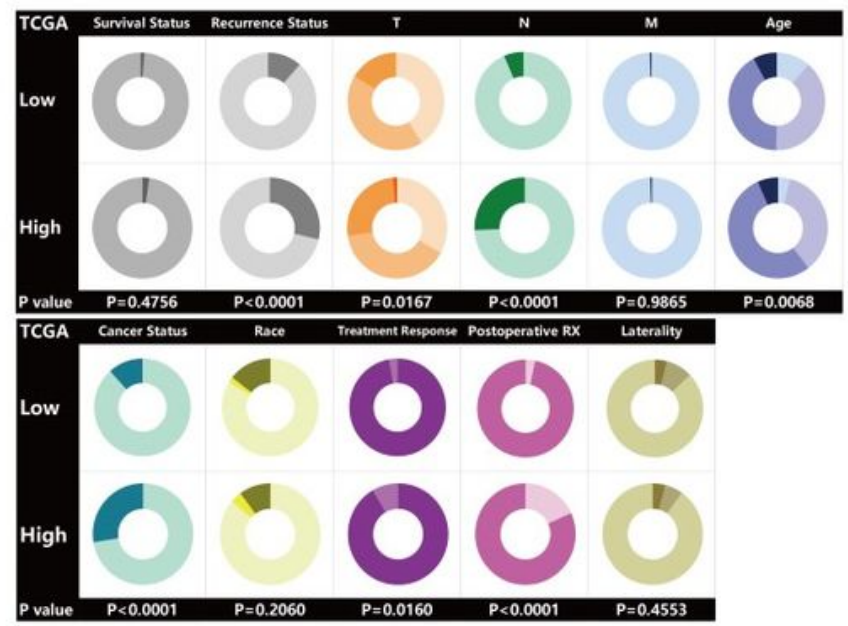

H

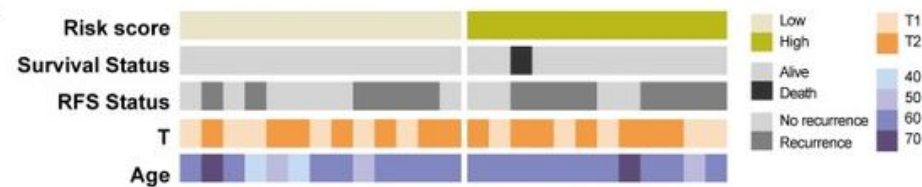

I

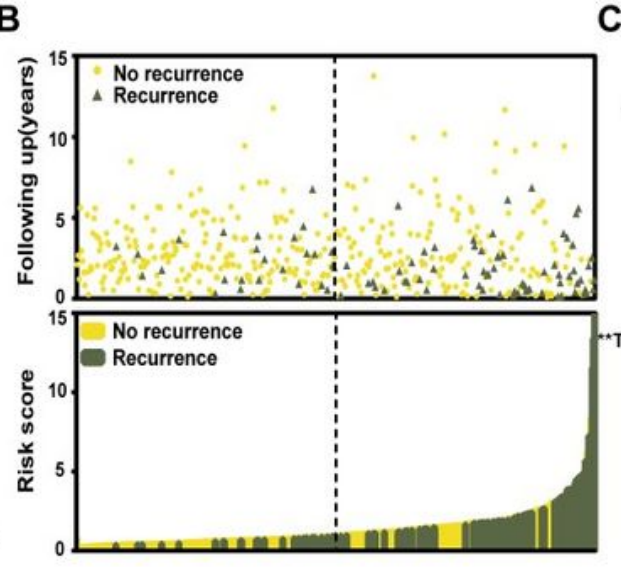

C

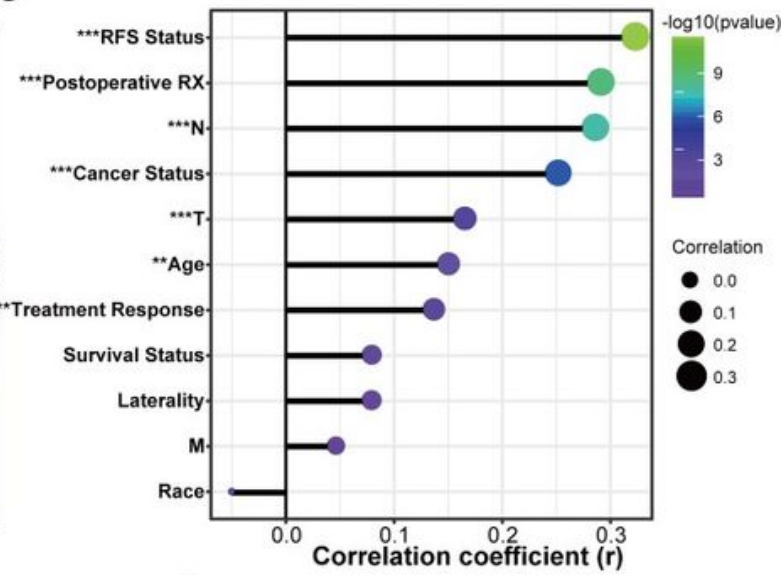

F

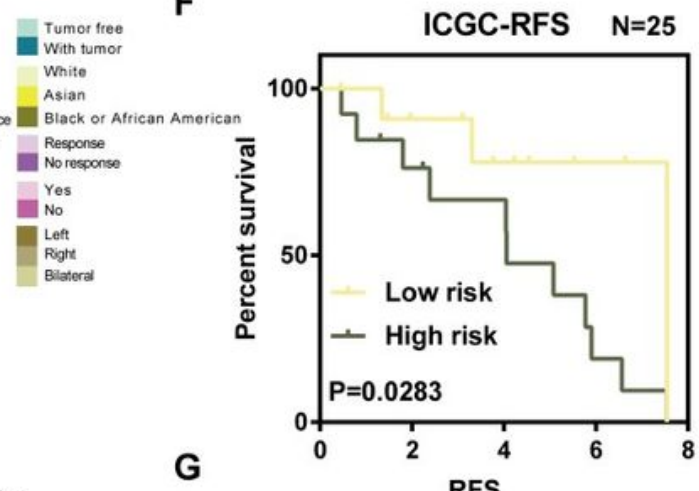

G
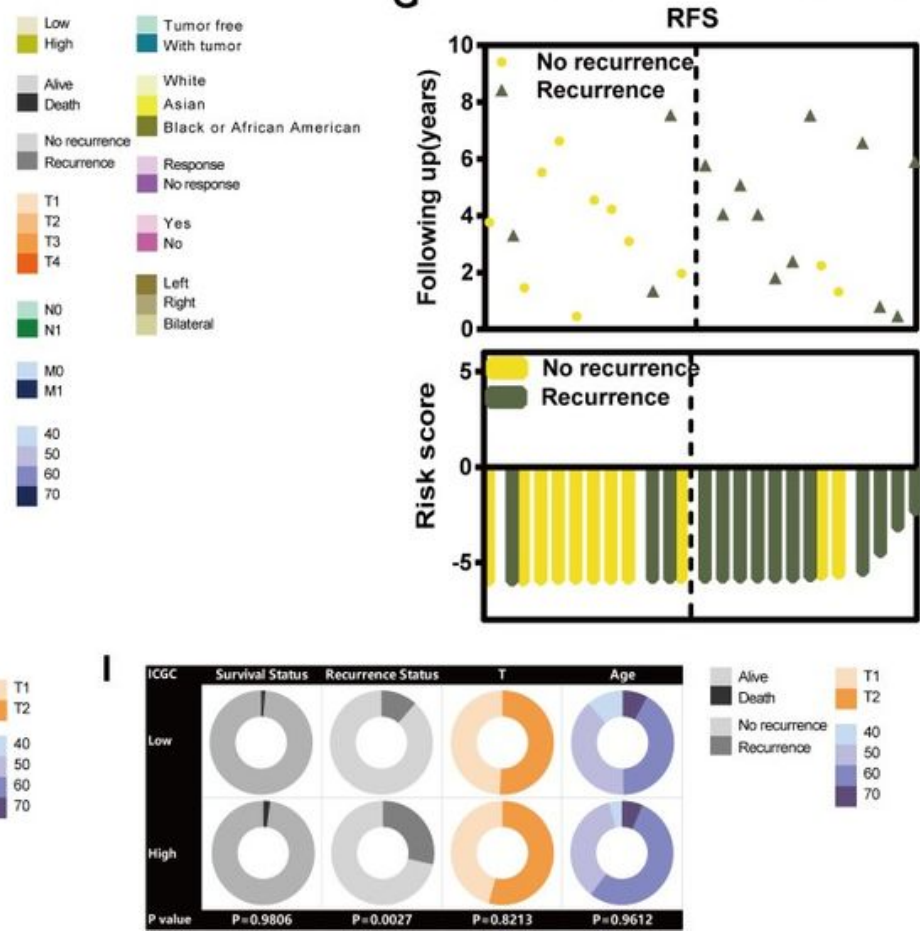
The TILTregSig can be served as a biomarker for RFS of prostate cancer patients in both TCGA and ICGA datasets. (A) Kaplan-Meier curves of RFS of low- and high- risk groups stratified by the TILTregSig in prostate cancer patients in TCGA datatset. (B) The distribution of gene risk scores and patients' recurrence status for prostate cancer patients in TCGA datatset. (C) Correlation analysis between risk score and clinical characteristics in prostate cancer. R: Spearman's correlation coefficient. (D) The strip chart of risk score and clinical characteristics for patients with prostate cancer in TCGA datatset. (E) Pie charts showing the Chi-squared test of clinicopathologic factors for low- and high- risk groups in prostate cancer samples from TCGA dataset. (F) Kaplan-Meier curves of RFS of low- and high- risk groups stratified by the TILTregSig in prostate cancer patients in ICGC dataset. (G) The distribution of gene risk scores and patients' recurrence status for prostate cancer patients in ICGC dataset. $(H)$ The strip chart of risk score and clinical characteristics for patients with prostate cancer in ICGC datatset. (I) Pie charts showing the Chi-squared test of clinicopathologic factors for low- and high- risk groups in prostate cancer samples from ICGC dataset. 


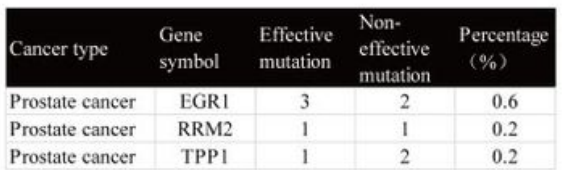

RRM2 : [Somatic Mutation Rate: $0.2 \%$ ] NM 001165931

ribonucleotide reductase M2 polypeptide isoform 2

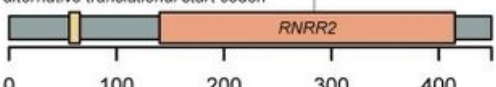

Missense_Mutation Frame_Shift_Ins

C
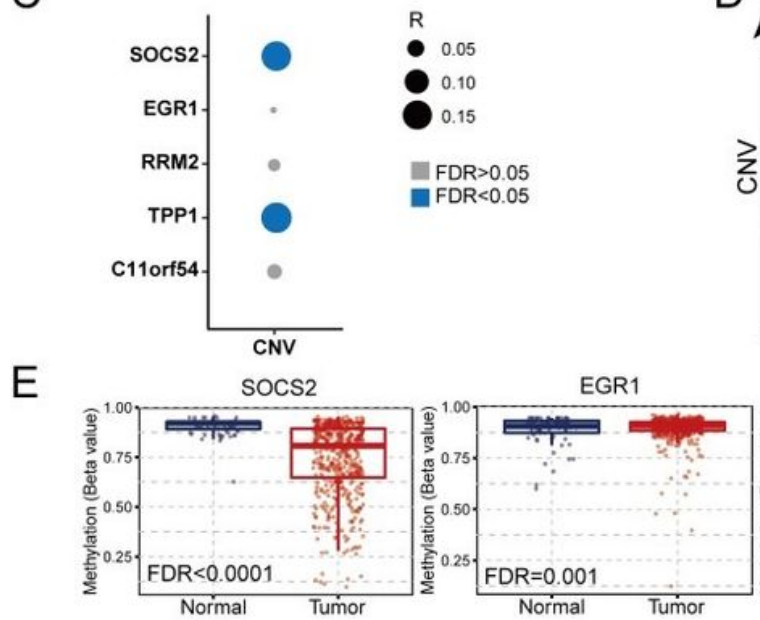
NM_000391

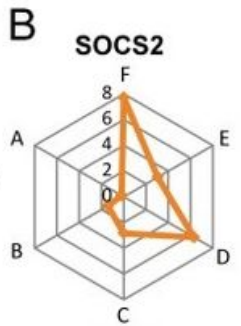

TTP1

EGR1 : [Somatic Mutation Rate: $0.6 \%$ ]

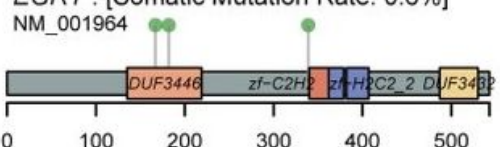

TPP1 : [Somatic Mutation Rate: $0.2 \%]_{\varphi}$
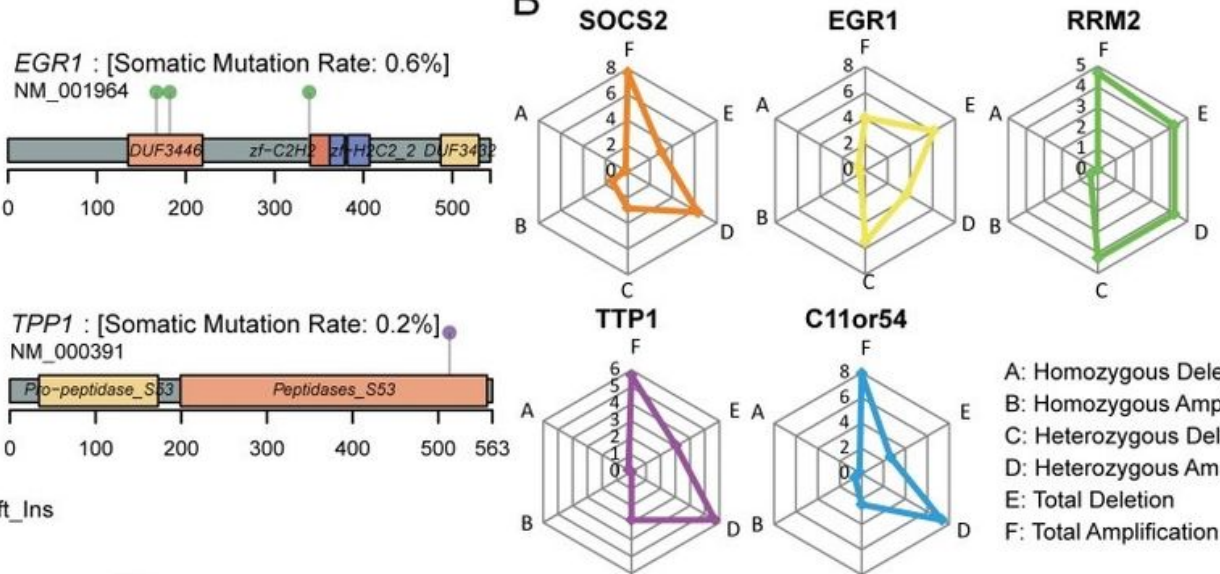

C11or54

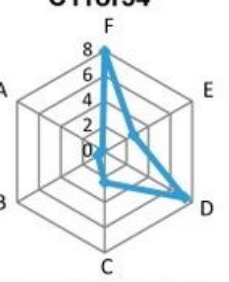

A: Homozygous Deletion B: Homozygous Amplification C: Heterozygous Deletion D: Heterozygous Amplification E: Total Deletion

F: Total Amplification
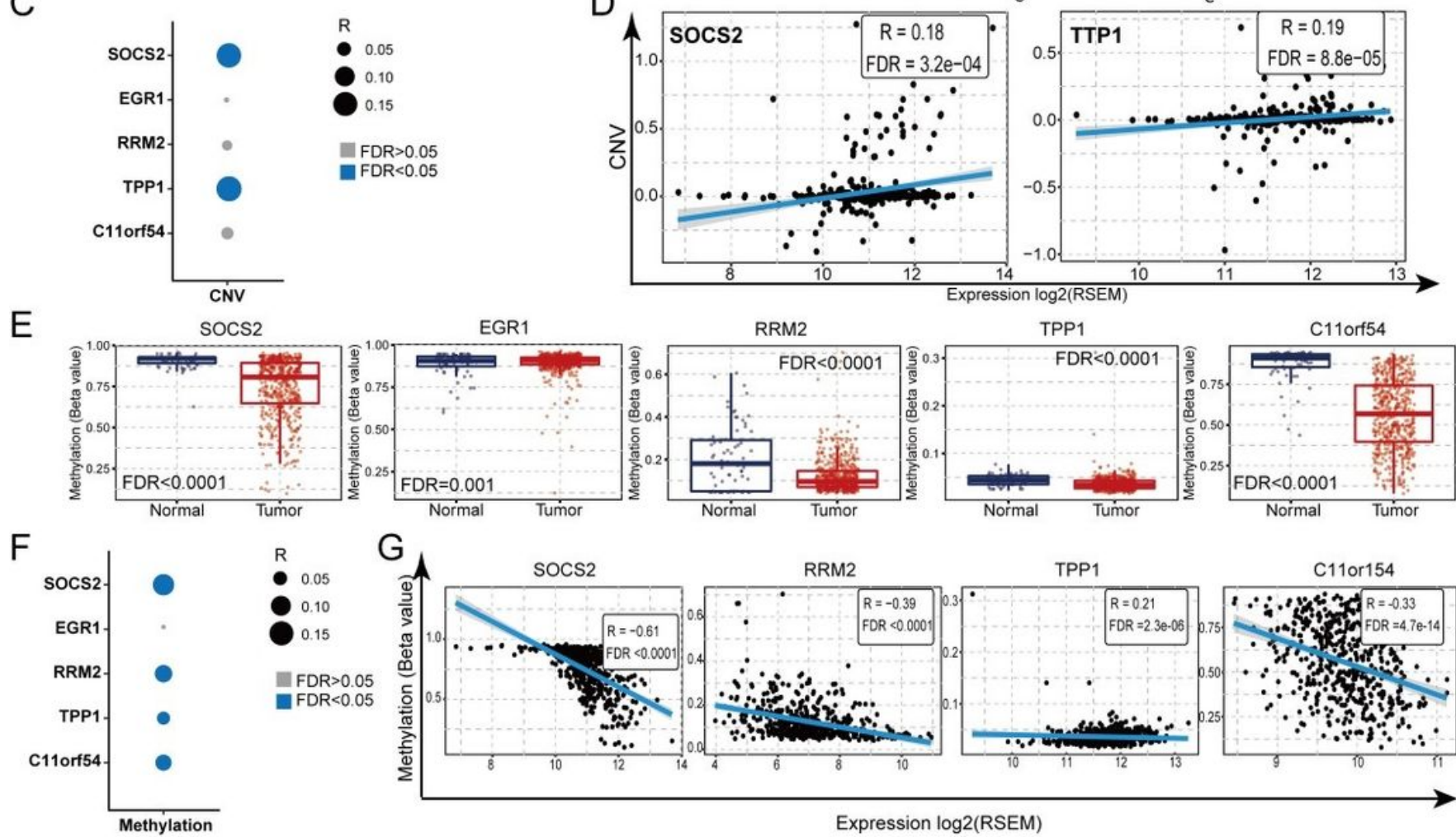

Expression log2(RSEM)

\section{Figure 2}

Gene alteration and methylation landscape of the five genes in the TILTregSig in prostate cancer. (A, B) The SNV status of the five genes in the TILTregSig of prostate cancer patients in GSCALite. (C) The correlation between CNV and the expression levels of the five genes in the TILTregSig of prostate cancer patients in GSCALite. (D) The correlation between CNV and the expression levels of SOCS2 and TPP1. R: Spearman's correlation coefficient. FDR: false discovery rate. $(E)$ The difference of gene methylation between normal and prostate cancer samples. (F) The correlation between gene methylation and the expression levels of the five genes in the TILTregSig of prostate cancer patients in GSCALite. (G) The correlation between gene methylation and the expression levels of SOCS2, RRM2, TPP1 and C11orf54. R: Spearman's correlation coefficient. FDR: false discovery rate. 
A
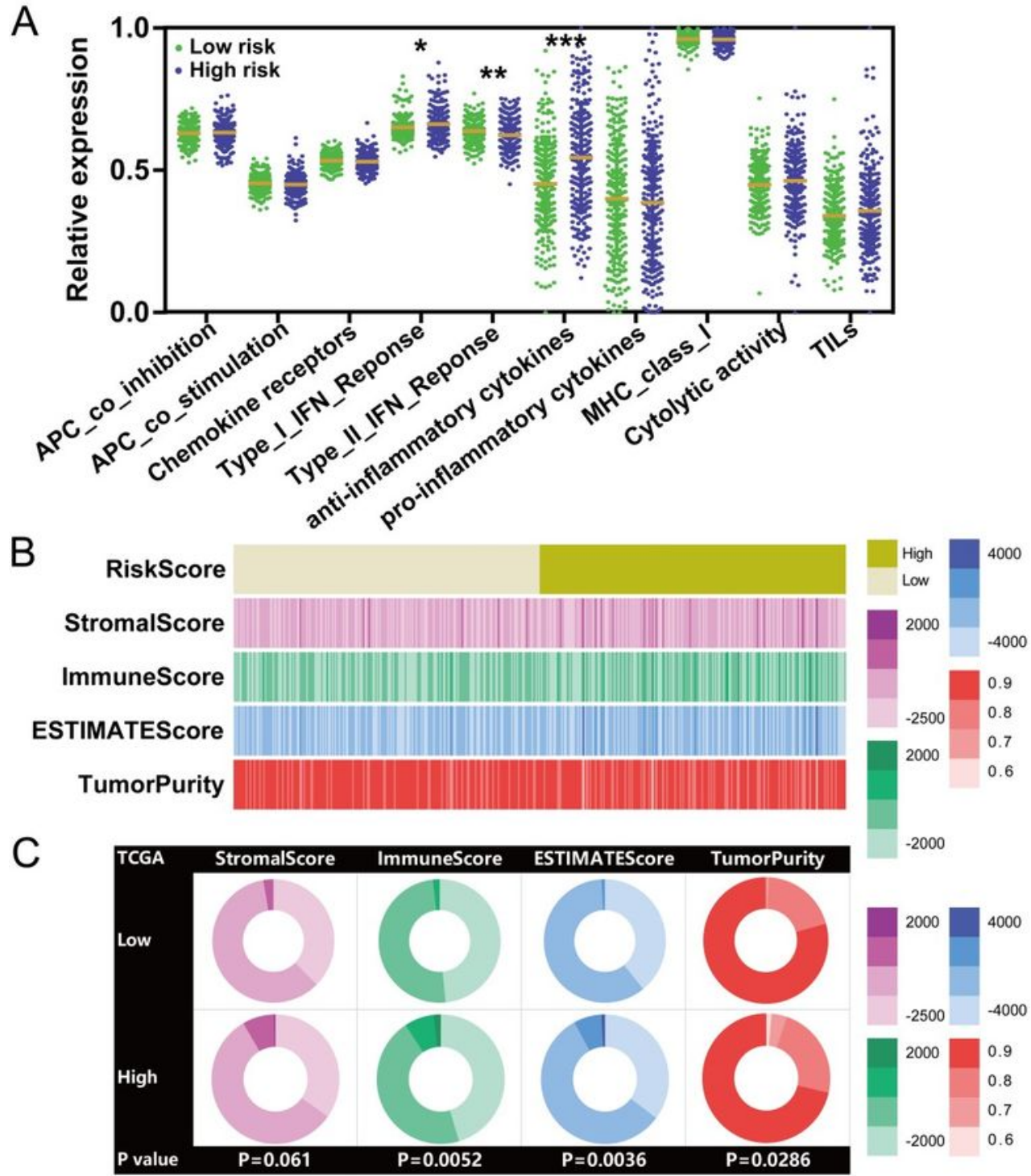

D

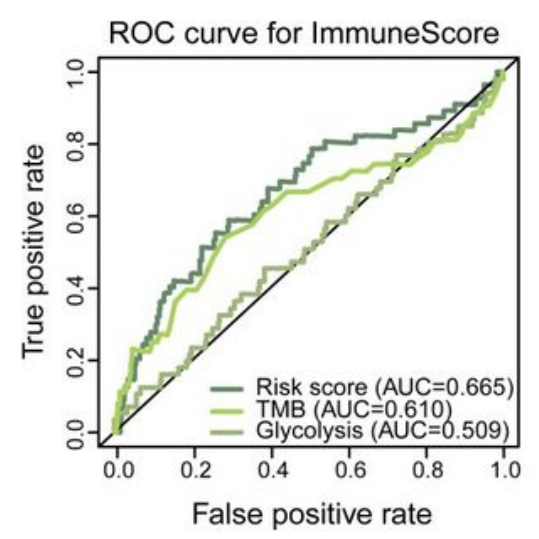

E

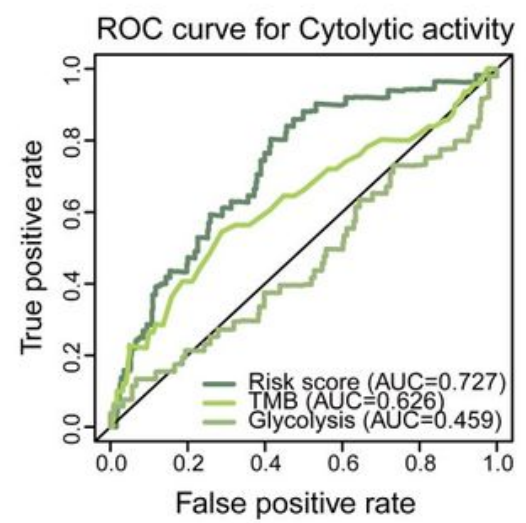

Figure 3

The TILTregSig shows stronger predictive ability for tumor immunity in prostate cancer. (A) The expression levels of immune-related signatures in low- and high- risk groups stratified by the TILTregSig in prostate cancer from TCGA dataset. (B) The strip chart of risk score, immune score, stromal score, ESTIMATE score and tumor purity of prostate cancer patients in TCGA datatset. (C) Pie charts showing the Chi-squared test of risk score, immune score, stromal score, ESTIMATE score and tumor purity for low- 
and high- risk groups in prostate cancer samples from TCGA dataset. Comparison of predictive ability of the TILTregSig, TMB and glycolytic activity for immune score (D) and immune cytolytic activity (CYT) (E) using ROC curves analysis.

A
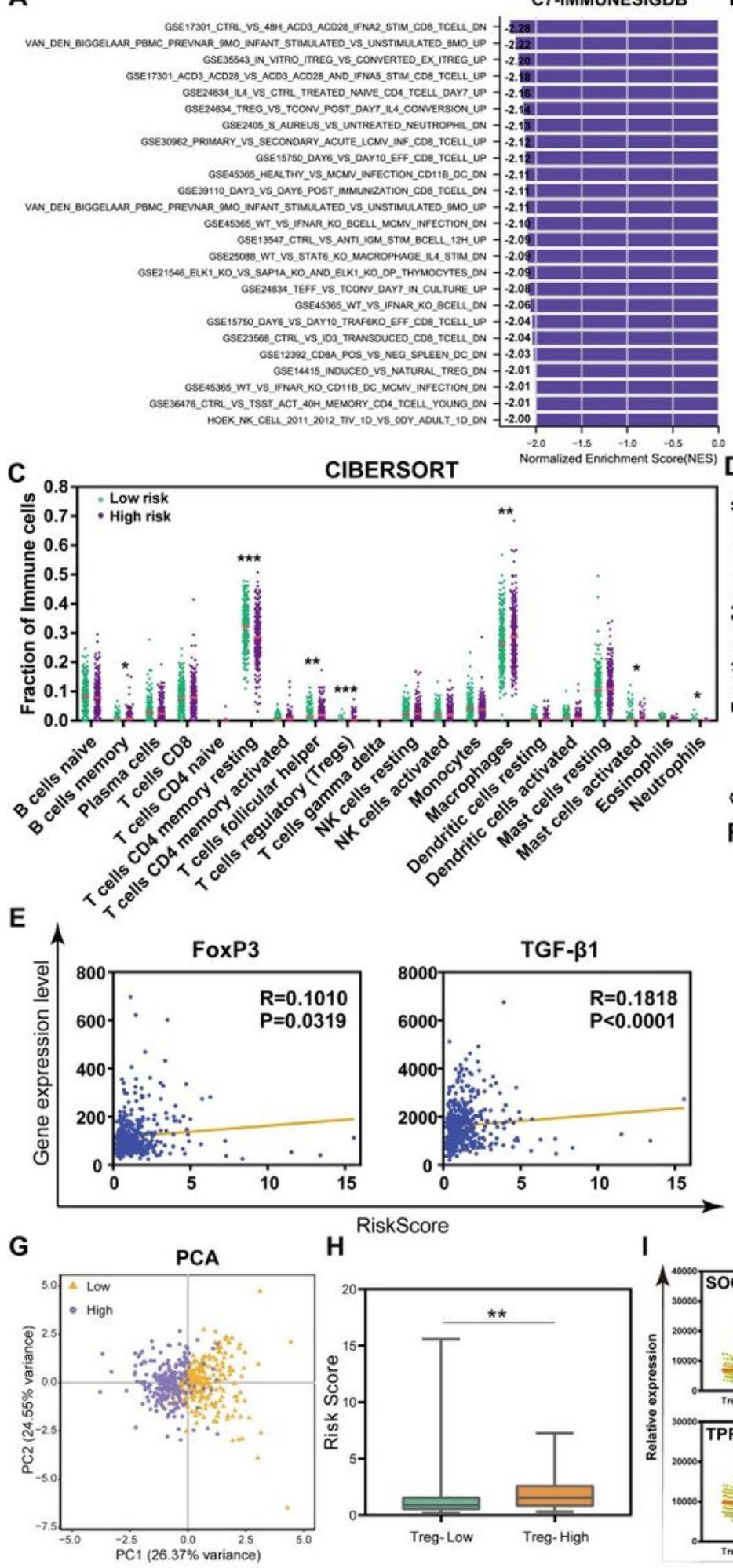

$\mathbf{H}^{\text {RiskScore }}$
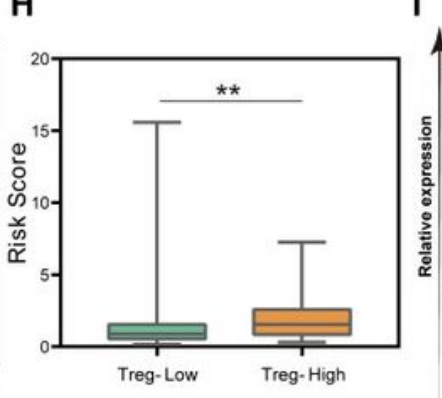

B

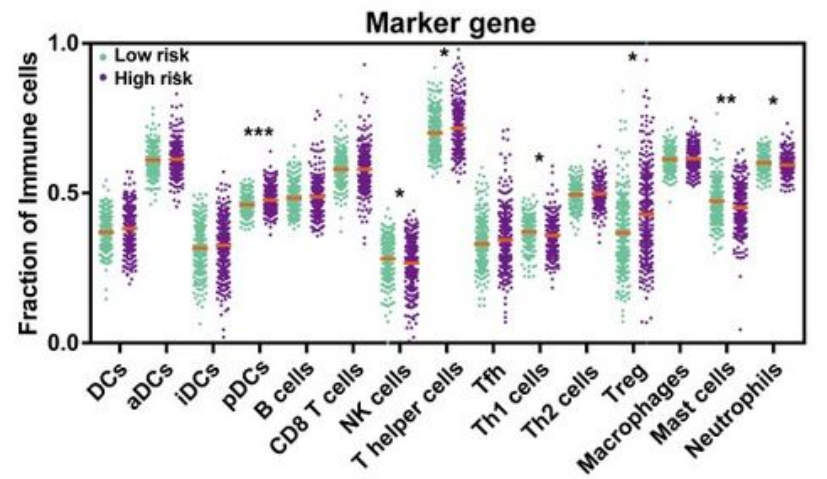

ImmuneCell AI
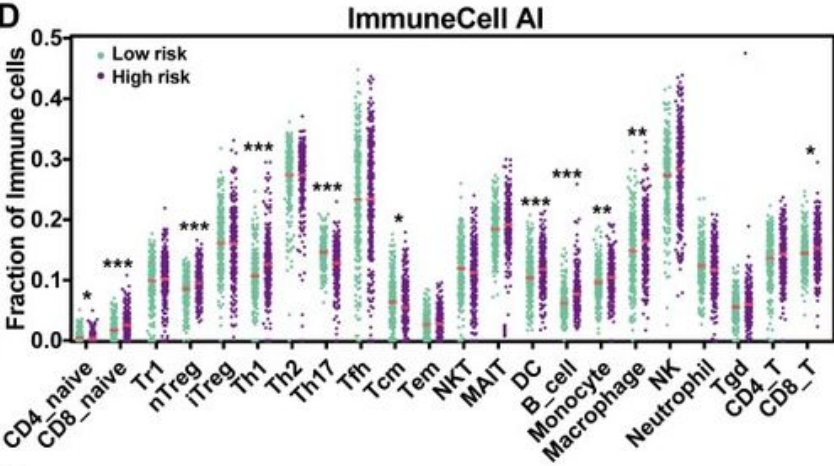

$\mathbf{F}$

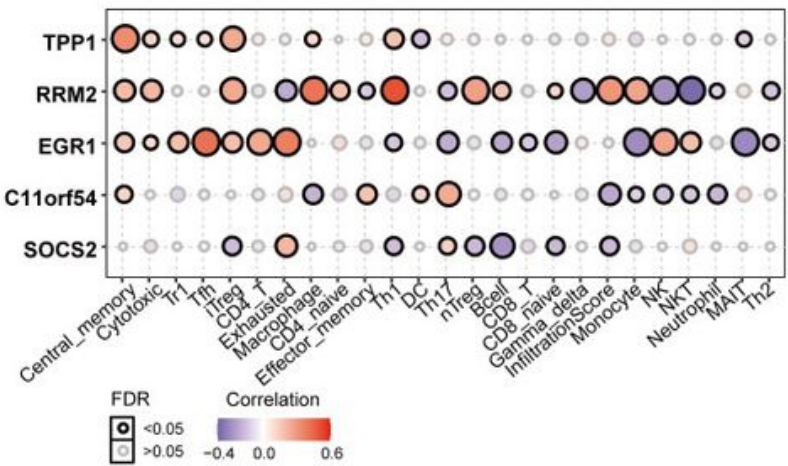

\section{Figure 4}

The TILTregSig can be served as a predictor for Tregs in prostate cancer. (A) GSEA analysis of the TILTregSig using the gene set of "c7.all.v7.4.symbles.gmt [immunologic signature]". The infiltrations of 
immune cells in high- and low- risk groups stratified by the risk score in prostate cancer samples from TCGA dataset using marker genes' expression analysis (B), CIBERSORT algorithm (C) and ImmuneCell Al database (D). (E) The correlation of the TILTregSig with FoxP3 and TGF- $\beta 1$ expression. (F) The correlation of the five genes in the TILTregSig with tumor infiltrating immune cells in prostate cancer samples from TCGA. (G) Principal components analysis (PCA) of the risk scores between Treg-low and Treg-high samples in prostate cancer. The distribution of the risk scores $(\mathrm{H})$ and the five genes expression (I) among samples grouped by infiltrations of Tregs in prostate cancer. $(\mathrm{J})$ Comparison of predictive ability of the TILTregSig and FoxP3 for Tregs infiltration using ROC curves analysis.

A

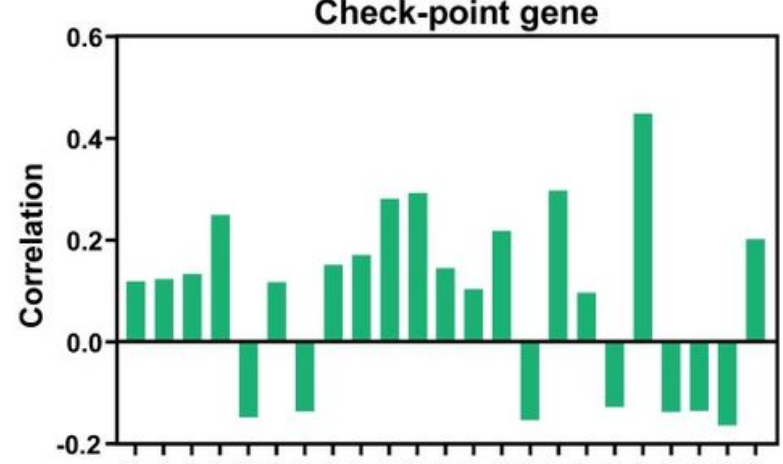

Check-point gene

B
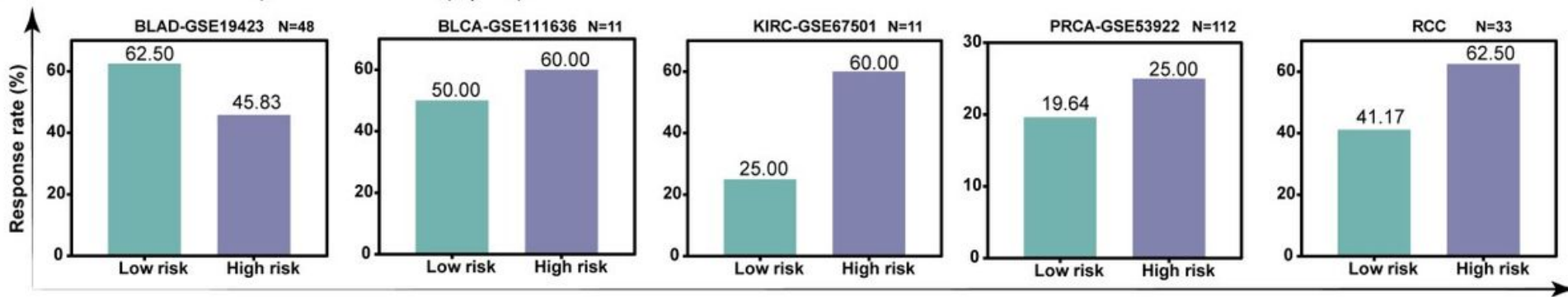

C

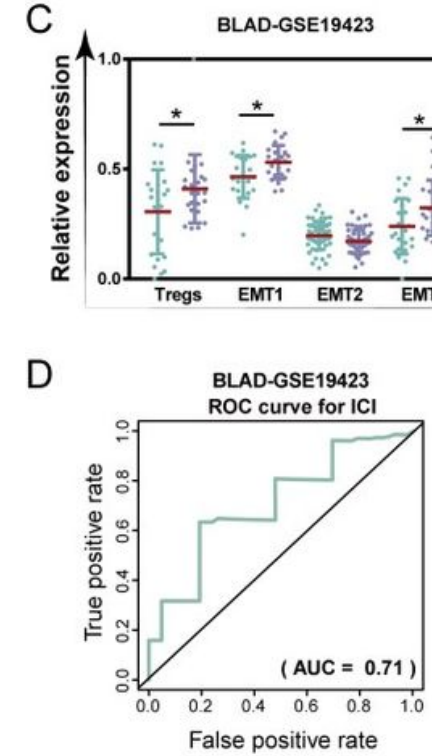

BLCA-GSE111636

KIRC-GSE67501

PRCA-GSE53922

RCC

\begin{tabular}{|l|c|c|c|c|c|}
\hline Gene & $\mathrm{R}$ & $\mathrm{P}$ & Gene & $\mathrm{R}$ & $\mathrm{P}$ \\
\hline LAG3 & 0.119 & 0.011 & CD27 & 0.104 & 0.026 \\
\hline CTLA4 & 0.124 & 0.008 & LGALS9 & 0.218 & $<0.0001$ \\
\hline CD80 & 0.134 & 0.004 & CD28 & -0.153 & 0.001 \\
\hline CD70 & 0.250 & $<0.0001$ & TNFRSF25 & 0.298 & $<0.0001$ \\
\hline TNFSF9 & -0.148 & 0.002 & ADORA2A & 0.097 & 0.039 \\
\hline PDCD1 & 0.118 & 0.012 & CD160 & -0.128 & 0.006 \\
\hline TNFSF15 & -0.136 & 0.004 & TNFRSF18 & 0.449 & $<0.0001$ \\
\hline TNFRSF14 & 0.152 & 0.001 & BTNL2 & -0.137 & 0.003 \\
\hline IDO2 & 0.171 & $<0.0001$ & TNFSF4 & -0.135 & 0.004 \\
\hline CD276 & 0.282 & $<0.0001$ & CD200 & -0.164 & $<0.0001$ \\
\hline TNFRSF4 & 0.293 & $<0.0001$ & NRP1 & 0.202 & $<0.0001$ \\
\hline HAVCR2 & 0.145 & 0.002 & & & \\
\hline
\end{tabular}

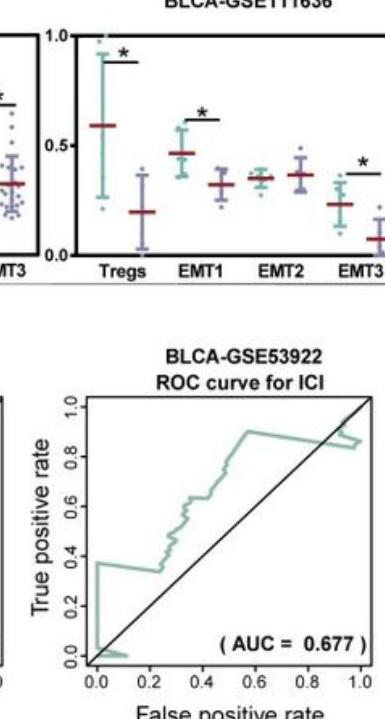

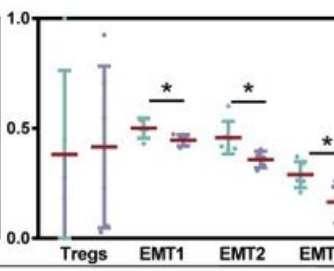
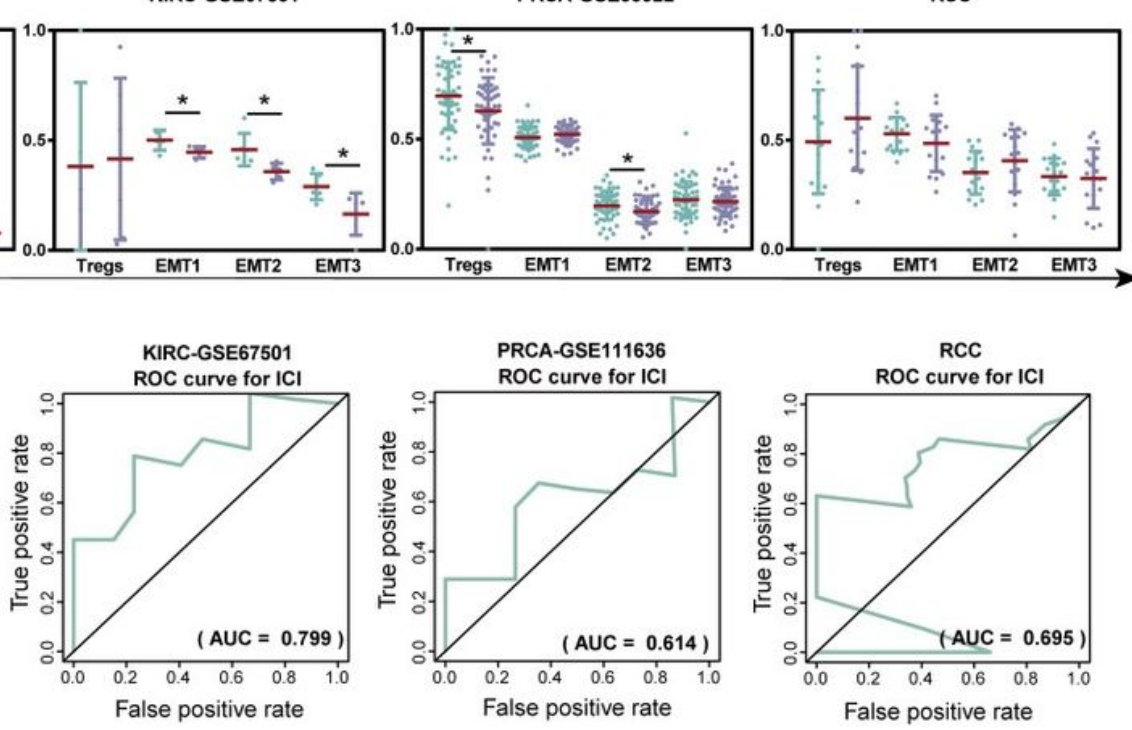

Figure 5

The TILTregSig is a potential predictor for immunotherapy response in prostate cancer. (A) The correlation of the risk scores with the expression levels of check-point genes in prostate cancer samples 
from TCGA datatset. R: Spearman's correlation coefficient. (B) The response rate to immunotherapy in low- and high- risk groups stratified by risk scores in each dataset. (C) The distribution of the Tregs infiltration and EMT activity among samples in low- and high- risk groups in each dataset. (D) ROC curves of the TILTregSig in predicting immunotherapy response in each dataset.

A

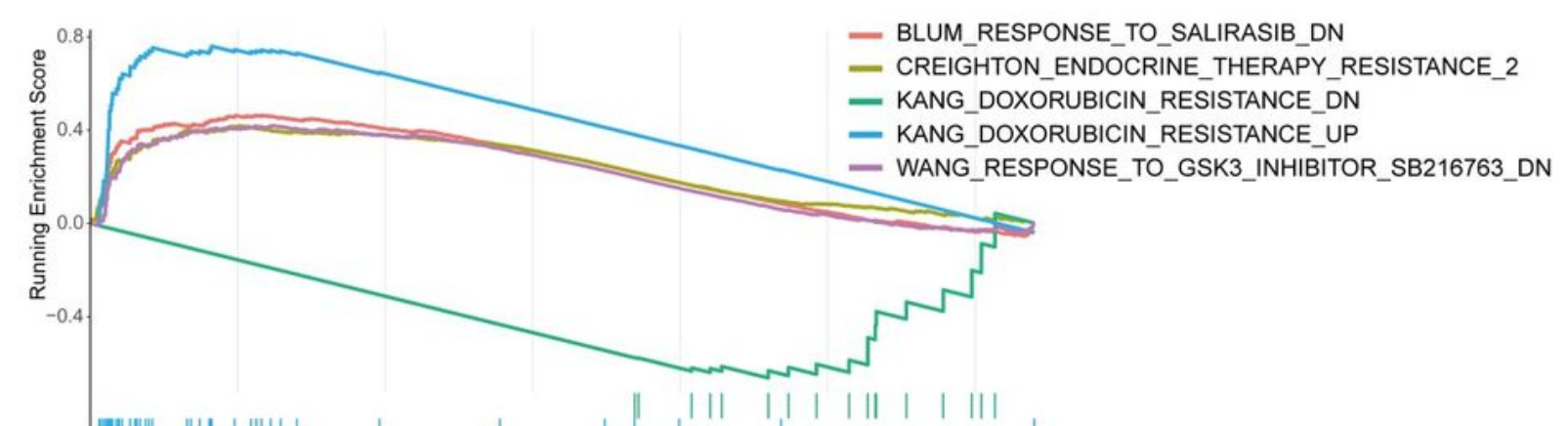

B

B Correlation between CTRP drug sensitivity and mRNA expression
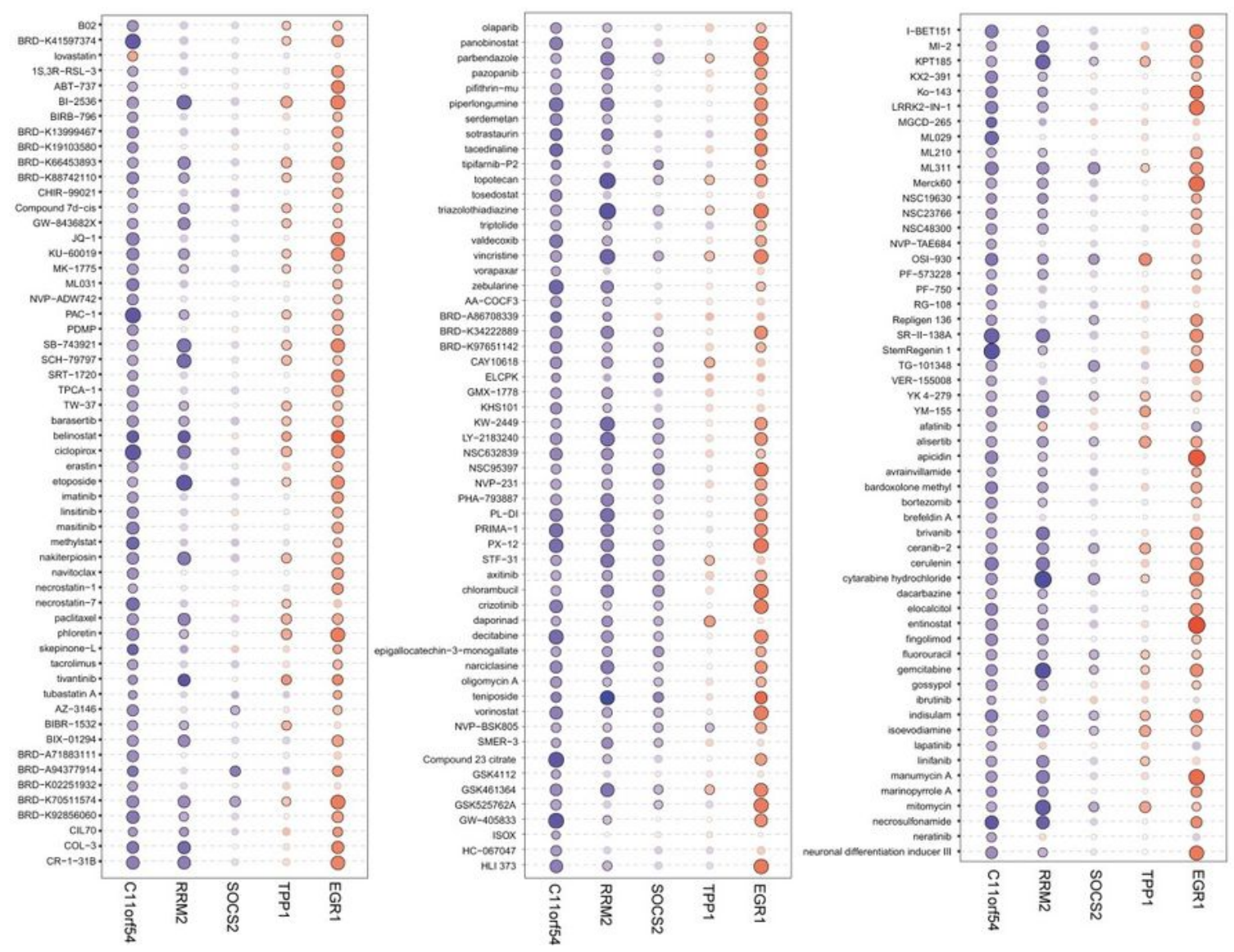

FDR

의 $<0.05$

Correlation $-0.3$

0.0

0.3

Figure 6 
The TILTregSig is a promising marker of cancer therapeutic resistance. (A) The relationship between the TILTregSig and therapeutic resistance using GSEA analysis. (B) A landscape plot was generated to depict the relationships between IC50 data of different molecules and the five genes expression profiles in cancers using GSCALite.

\section{Supplementary Files}

This is a list of supplementary files associated with this preprint. Click to download.

- FigureS1.tif

- Figures2.tif

- FigureS3.tif

- Figures4.tif

- SupplementaryTable.xlsx 